\title{
1 A multispecies amplicon sequencing approach for genetic diversity assessment in 2 grassland plant species
}

\section{Authors and affiliations}

4 Miguel Loera-Sánchez

5 Molecular Plant Breeding, Institute of Agricultural Sciences, ETH Zurich, Universitaetstrasse 2,

68092 Zurich, Switzerland

7 Bruno Studer

8 Molecular Plant Breeding, Institute of Agricultural Sciences, ETH Zurich, Universitaetstrasse 2,

98092 Zurich, Switzerland

10 Roland Kölliker

11 Molecular Plant Breeding, Institute of Agricultural Sciences, E'TH Zurich, Universitaetstrasse 2,

128092 Zurich, Switzerland. Email: roland.koelliker@usys.ethz.ch 


\section{Abstract}

14 Grasslands are widespread and economically relevant ecosystems at the basis of sustainable 15 roughage production. Plant genetic diversity (PGD; i.e., within-species diversity) is related to 16 many beneficial effects to the ecosystem functioning of grasslands. The monitoring of PGD in 17 temperate grasslands is complicated by the multiplicity of species present and by a shortage of

18 methods for large-scale assessment. However, the continuous advancement of high-throughput

19 DNA sequencing approaches have improved the prospects of broad, multispecies PGD 20 monitoring. Among them, amplicon sequencing stands out as a robust and cost-effective 21 method.

22 Here we report a set of twelve multispecies primer pairs that can be used for high-throughput 23 PGD assessment in multiple grassland plant species. The loci targeted by the amplicons were 24 selected and tested in two phases: a "discovery phase" based on a sequence capture assay (611 target nuclear loci assessed in 16 grassland plant species), which resulted in the selection of eleven loci; and a "validation phase", in which the selected loci were targeted and sequenced using twelve multispecies primers in test populations of Dactylis glomerata L., Lolium perenne L., Festuca pratensis Huds., Trifolium pratense L. and T. repens L. The resulting multispecies amplicons had overall nucleotide diversities per species ranging from $5.19 \times 10^{-3}$ to $1.29 \times 10^{-2}$, which is in the range of flowering-related genes but slightly lower than pathogen resistance genes. We conclude

31 that the methodology, the DNA sequence resources, and the amplicon-specific primer pairs 32 reported in this study provide the basis for large-scale, multispecies PGD monitoring in grassland 33 plants. 


\section{Introduction}

35 Grasslands cover an estimated $40 \%$ of Earth's land, fulfilling many ecosystem services including

36 the preservation of soil integrity and regulation of water, carbon and nitrogren flows (Bengtsson

37 et al., 2019; Reynolds, 2005; Zhao, Liu, \& Wu, 2020). Multispecies grasslands are important

38 sources of roughage for ruminant livestock and provide the basis for sustainable meat and dairy

39 production (Zhao et al., 2020). They are also of unique cultural relevance and serve as places of

40 recreation (Huber \& Finger, 2020; Zhao et al., 2020). Plant biodiversity, including genetic

41 diversity (i.e., within-species diversity), plays an important role in grassland ecology. Recent work

42 has shown that high levels of plant genetic diversity in grasslands confer population resistance

43 against invasive plants (Hadincová et al., 2020) and increase yield stability under environmental

44 stress conditions, in part due to interactions between plant species richness and genetic diversity

45 (Malyshev et al., 2016; Meilhac et al., 2019; Prieto et al., 2015). Furthermore, valuable genetic

resources for forage breeding are to be found along the wide geographical range for grasses

47 (Poaceae) and legumes (Fabaceae), the two most economically relevant families of forage crop

species found in grasslands. Nevertheless, compared to species richness, genetic diversity is still

underexplored for most taxonomic groups in all domains of life, including many grass and

legume species found in semi-natural grasslands of temperate regions. This is mainly because

most traditional assessment methods are time- and resource-demanding, particularly for constant and broad monitoring, which is the aim of current international biodiversity protection initiatives, such as the Convention of Biological Diversity of the United Nations (Hoban et al., 2020; Laikre et al., 2020; Pärli et al., 2021).

Recent advances in high-throughput sequencing technologies are rapidly improving this scenario.

56 Such technologies have enabled genetic diversity assessments in non-model organisms, including

57 the forage crop species from temperate regions (Loera-Sánchez, Studer, \& Kölliker, 2019). 
58 Compared to traditional methods for genetic diversity assessment (e.g., with SSRs), hundreds of

59 samples and tens of thousands of markers may be processed simultaneously with high-

60 throughput sequencing approaches. High-throughput approaches suitable for genetic diversity

61 assessment in non-model organism include complexity reduction methods (e.g., genotyping-by-

62 sequencing [GBS] and restriction site-associated DNA sequencing [RADseq]) and target enrichment methods (e.g., sequence capture and amplicon sequencing. Those methods differ in the amount of DNA they require, the marker density (i.e., the number of SNPs) they produce and the costs they imply (Carroll et al., 2018; Harvey et al., 2016).

The choice of a method to assess genetic diversity in grassland plant species in a large scale would require making a balance of its technical features along with the biological features of grassland plants. Grassland plant species often display high levels of intrapopulation genetic diversity and low levels of interpopulation differentiation, which is characteristic of outbreeding species (Hamrick \& Godt, 1996). This is the case for many important forage crops, including ryegrasses (Lolium spp.), fescues (Festuca spp.), orchardgrass (Dactylis glomerata L.) and clovers (Trifolium spp.;

72 (Collins et al., 2012; Cuyeu et al., 2013; Last et al., 2013; Liu et al., 2018). Because of such high levels of genetic diversity, large samples per population are usually necessary to estimate genetic diversity in these species (Kölliker et al., 2009). However, likely because of such high levels of diversity, a high marker density is not necessary to detect genetic differentiation in populations of such taxa. This has been observed in Lolium perenne L. (Liu et al., 2018), as well as in other highly diverse species, such as open-pollinated maize (Zea mays L.) landraces (Caldu-Primo et al., 2017) and Arabidopsis halleri, (L.) O'Kane \& Al-Shehbaz, an outbreeding model species (Fischer et al., 2017).

In this study, we present a novel genetic diversity assessment approach based on amplicon sequencing that can be used in multiple forage grass and legume species. Taking advantage of the 
82 naturally high levels of genetic diversity and low levels of linkage disequilibrium of such

83 outcrossing species, we hypothesized that a reduced set of nuclear loci would contain enough

84 polymorphisms for genetic diversity analysis. We describe the steps we took to find such loci in

85 sixteen forage crop species from sub-alpine grasslands (the "discovery" phase of this study) and

86 how we evaluated them in test populations of five of such species, including pooled-plant

87 samples (the "validation" phase). We then discuss the prospects and improvement avenues for

88 genetic diversity assessment in grassland plants based on multispecies amplicon sequencing. 


\section{Materials and Methods}

90 The development of multispecies amplicons for genetic diversity assessments in grassland plant

91 species followed a two-phase approach. In the "discovery phase", the genetic diversity of 611

92 conserved loci was assessed in 16 forage species with targeted sequencing. Then, in the

93 "validation phase", selected multispecies amplicons were sequenced in test populations of five

94 species (Figure 1).

95 Discovery phase

96 Bait design and synthesis

97 A set of 734 putatively single-copy, orthologous genes (SCOGs) was identified with OrthoMCL

98 (L. Li, Stoeckert Jr., \& Roos, 2003) in selected reference genomes including Arabidopsis thaliana

99 (L.), Heynh. (GCA_000001735.1; Lamesch et al., 2012), Brachypodium distachyon (L.) P.Beauv.

100 (GCA_000005505.4; Vogel et al., 2010), Glycine max (L.) Merr., 1917(GCA_000004515.4;

101 Schmutz et al., 2010), Solanum lycopersicum L. (GCA_000188115.3; Sato et al., 2012), Theobroma

102 cacao (L.) (GCA_000403535.1; Motamayor et al., 2013), Trifolium pratense L. (GCA_900079335.1;

103 De Vega et al., 2015)and Vitis vinifera L. (GCA_000003745.2; Jaillon et al., 2007). The reference

104 genomes were downloaded from EnsemblPlants (https://plants.ensembl.org/index.html). An

105 additional genome assembly of Lolium perenne L. (GCA_001735685.1) was obtained from NCBI

106 (https://www.ncbi.nlm.nih.gov). Bait design was performed using BaitFisher v1.2.7 (Mayer et al.,

107 2016) and non-continuous multiple sequence alignments of the 734 SCOGs as input. The

108 annotation of $A$. thaliana (GCA_000001735.1) was used to account for intron-exon boundaries.

109 Additionally, 1,277 Ultra Conserved-Like Elements (ULEs) were identified with Phyluce v1.4

110 (Faircloth, 2016). For this purpose, the same set of genomes mentioned above was

111 complemented with Aegilops tauschii Coss. (GCA_002575655.1; Luo et al., 2017), Leersia perrieri

112 (A.Camus) Launer (GCA_000325765.3), Lotus japonicus (Regel) K.Larsen, 1955 
113 (GCA_000181115.2; Sato et al., 2008), Medicago truncatula Gaertn. (GCA_000219495.2; Tang et

114 al., 2014), Oryza sativa L. subsp. japonica (GCA_001433935.1; Kawahara et al., 2013) and Phaseolus

115 vilgaris L., 1753 (GCA_000499845.1; Schmutz et al., 2014). The Phyluce UCE-identification

116 pipeline was performed thrice using one of the following guiding genomes in each iteration: $A$.

117 thaliana (GCA_000001735.1), B. distachyon (GCA_000005505.4) and $M$. truncatula

118 (GCA_000219495.2). Phyluce v1.4 was then used to find candidate bait sequences.

119 To control which genomic regions were targeted, the bait sequences were mapped to three

120 annotated reference genomes: A. thaliana (GCA_000001735.1), B. distacbyon (GCA_000005505.4)

121 and $M$. truncatula (GCA_000219495.2). Bait sequences were used to synthesize a custom

122 myBaits ${ }^{\circledR}$ kit (Arbor Biosciences, MI, USA) from now on referred to as the "FORAGE-611"

123 baits. 
126 Seeds of 16 forage species were germinated on filter paper and their seedlings were transferred

127 into pot trays (77 wells, $50 \mathrm{~cm} \times 32 \mathrm{~cm}$, with compost as substrate). The 16 species were: Alopecumus pratensis L., Arrhenaterum elatius L., Cynosums cristatus L., Dactylis glomerata L., Festuca

136 Germany) and its integrity visually inspected by agarose gel electrophoresis $(1 \% \mathrm{w} / \mathrm{v})$. DNA

137 purity and concentration were determined with a NanoDrop ${ }^{\mathrm{TM}}$ spectrophotometer

138 (ThermoFisher Scientific, Waltham, MA, USA).

140 Dual-indexed libraries were constructed using the NEBNext ${ }^{\circledR}$ Ultra ${ }^{\mathrm{TM}}$ II DNA Library Prep Kit 141 for Illumina (New England Biolabs, UK). Each species was represented by six libraries: five 142 single-plant libraries and one pooled DNA library, for a total of 96 libraries. The pooled-plant 143 libraries consisted of equimolarly pooled DNA from the 5 single-plants. The average library 144 insert size was $\sim 550 \mathrm{bp}$.

145 After indexing, the libraries were divided in four pools of $6 \times$ libraries, four pools of $8 \times$ libraries 146 and four pools of $10 \times$ libraries. Each pool was hybridized to the FORAGE-611 baits. The target 147 DNA fragments were enriched following manufacturer instructions. Fragments were sequenced 148 using the Illumina MiSeq v3 Reagent Kit (2 x 300 bp, 600 cycles). The raw pair-ended reads were 
149 merged using BBMerge (Brian Bushnell, Rood, \& Singer, 2017) with default parameters. Adapter

150 removal and quality filtering was done using fastp (Chen et al., 2018) with default parameters on 151 the merged reads.

152 Sequencing quality control

153 The pooled DNA libraries were used to construct pseudo-reference assemblies (pseudo-RAs) 154 using SPAdes (Bankevich et al., 2012). One pseudo-RA was assembled for each species. The 155 sequences of the FORAGE-611 baits were mapped to the pseudo-RAs using BBMap (B. 156 Bushnell, 2014) with default parameters. Bait-mapping coordinates were determined with 157 SAMtools (H. Li, 2011; H. Li et al., 2009) and BEDtools (Quinlan \& Hall, 2010). Target locus 158 centers were defined as the middle-point between the 3'-most and 5'-most coordinate of the 159 mapped bait sequences that belong to the same locus. A target locus region was defined as the 160 sequences spanning 500 bp up- and downstream from each locus center. In the cases where baits 161 mapped to more than one pseudo-RA contig, only the largest contig was kept for further 162 analysis.

163 The 80 single-plant libraries were mapped to their corresponding pseudo-RAs with Bbmap. 164 Reads mapping within target locus regions were considered as "on-target reads". Duplicate reads 165 were handled with Picard MarkDuplicates (Broad Institute, 2019), marking them for variant 166 calling and removing them for k-mer richness calculations.

167 A locus with >5 mapped reads was labelled as a quality-controlled locus (QCL). Libraries with $168>100$ QCL and >1,000 total on-target reads were labelled as quality-controlled libraries (QC-libs).

169 Only QC-libs and on-target reads were considered for further analysis. Read-mapping statistics 170 were determined using BEDtools. 


\section{Diversity metrics}

172 Four diversity metrics were used to rank the 611targeted loci within each species: nucleotide

173 diversity $(\pi)$, SNP-based nucleotide diversity, SNP density and normalized $k$-mer richness. Each

174 metric was calculated per locus within each species.

175 To calculate SNP-based within-species gene diversity, variant calling was done species-by-species

176 using BCFtools mpileup (H. Li, 2011; H. Li et al., 2009) on the BAM files from species with >3

177 QC-libs. The resulting variant call files (VCFs) were filtered using BCFtools for biallelic SNPs

178 with a minimum quality of 20 , a minimum allele frequency of 0.1 and a minimum read depth per

179 sample of 5. Estimations of $\pi$ were calculated per site using VCFtools, treating all samples as

180 diploid (Danecek et al., 2011). Locus-level estimations were then summed and divided by locus

181 length, which in turn were based on the scaffold lengths in pseudo-RA and had a maximum value

182 of $1 \mathrm{kbp}$ per locus. SNP count and SNP densities were calculated using the same custom R script

183 based on genotype tables produced with VCFtools.

$$
N K R=\frac{K_{c}}{L-k+1}
$$

To calculate normalized $k$-mer richness (NKR), de-replicated reads were extracted from dereplicated BAM files using SAMtools bam2fa, creating separate FASTQ files for each locus within each library. K-mers of $k=25$ in each FASTQ file were determined using jellyfish (Marçais \& Kingsford, 2011). K-mer dumps were filtered by discarding $k$-mers with less than $5 \mathrm{x}$ sequencing depth. Locus-specific NKR was calculated at the species level by concatenating the filtered $k$-mer dumps, counting unique $k$-mers within each concatenated dump and then dividing the value of such unique $k$-mer count by maximum expected $k$-mer count per locus $(L-k+1)$. This is summarized in equation 1 , where $K_{c}$ indicates the count of unique $k$-mers, $k$ indicates $k$ mer length and $L$ indicates locus length. 
194 Total diversity metrics were calculated for each species. Total NKR was calculated by dividing

195 the sum of all unique $k$-mers in all loci by the total expected $k$-mers in all loci $\left(\left(L_{\text {total }}-k+1\right.\right.$,

196 where $L_{\text {total }}$ is the sum of the lengths of all loci in base pairs units). Total SNP count is the sum of

197 all SNPs found in all loci and the SNP density per kbp was calculated following the formula:

$198 S N P_{\text {total }} \times 1,000 / L_{\text {total }}$, where $S N P_{\text {total }}$ is the total SNP count. Total $\pi$ was calculated using formula

1992 , where $\pi_{i}$ is the nucleotide diversity of the $i$-th locus, $L_{i}$ is the length of the $i$-th locus and $a$ is the

200 total number of loci.

$$
\frac{\sum_{i}^{a} \pi_{i} \times L_{i}}{L_{\text {total }}}
$$

\section{Primer design}

204 Eleven loci out of the 611 initially captured were selected for primer design. Selection criteria

205 included having high median gene diversity and normalized $k$-mer richness, as well as being

206 present in as many species as possible. Primers were designed using PriMux (Hysom et al., 2012)

207 with a target amplicon size of $500 \mathrm{bp}$ and primer size of 25 nt. Primers with successful in silico

208 and in vitro multispecies PCRs were selected for further testing. Thirteen primer pairs were

209 selected for synthesis (Microsynth AG, Balgach, Switzerland). Six primer pairs were grass-

210 specific, six were legume-specific and one pair was found in both plant families. Of those, a final

211 set of twelve primer pairs produced amplicons that were in distinct within each family.

\section{Validation phase}

214 For single-plant DNA extractions, seeds of five forage species (D. glomerata, F. pratensis, L. perenne,

215 T. pratense L. and T. repens) were germinated and used for DNA extraction after 3 to 4 weeks of 216 growth as described above. Each species was represented by 16 individual plants from three 
217 different cultivars (Supplementary Table 4). For pooled-plant DNA extractions, ground leaf

218 material from the same 16 plants per species was pooled at equal proportions (50 $\mathrm{mg}$ per plant),

219 mixed and $20 \mathrm{mg}$ of that mixture were used for DNA extraction. DNA was diluted to $5 \mathrm{ng} / \mu \mathrm{L}$.

Amplicon sequencing

221 Selected amplicons (Table 1) were amplified in $25 \mu \mathrm{L}$ PCR reactions containing $15 \mathrm{ng}$ of template

222 DNA, 1x flexi buffer (Promega, Madison, WI, USA), $2 \mathrm{mM} \mathrm{MgCl}_{2}, 200 \mu \mathrm{M}$ dNTPs, each primer

223 at $0.4 \mu \mathrm{M}$ and 0.75 units of Go'Taq ${ }^{\circledR}$ G2 Flexi DNA Polymerase (Promega, Madison, WI,

224 USA).PCR conditions were $5 \mathrm{~min}$ at $94^{\circ} \mathrm{C}$ followed by 25 cycles of $40 \mathrm{~s}$ at $94^{\circ} \mathrm{C}, 1 \mathrm{~min}$ at a 225 primer-specific melting temperature (Tm; Table 1) and $40 \mathrm{~s}$ at $72^{\circ} \mathrm{C}$, followed by a final extension 226 cycle of $10 \mathrm{~min}$ at $72^{\circ} \mathrm{C}$. The amplicons for each sample were then equimolarly pooled.

227 Dual-indexed libraries were constructed using the NEBNext ${ }^{\circledR}$ Ultra ${ }^{\mathrm{TM}}$ II DNA Library Prep Kit 228 for Illumina (New England Biolabs, UK). In total, 80 single-plant libraries (16 per species) and 15 229 pooled-plant libraries (three per species) were prepared. Dual-indexed libraries were equimolarly 230 pooled and sequenced using the Illumina MiSeq v3 Reagent Kit ( 2 x 300 bp reads; 600 cycles).

231 Merging of raw pair-ended reads, adapter removal and quality filtering were done as described 232 above. Additionally, to remove possible remnants of primer sequences, reads were trimmed 25 233 bp at each end using cutadapt (Martin, 2011).

\section{Diversity metrics}

235 To calculate SNP-based diversity metrics, quality-controlled reads were mapped to pseudo-

236 reference assemblies, which consisted only on the sequences of the selected loci taken from the 237 sequence capture data of the discovery phase. The pseudo-reference assemblies also contained 238 the sequences of DNA barcode $r b c I a$ corresponding to each species, which were obtained from 239 the Barcoding Of Life Datasystems (Ratnasingham \& Hebert, 2007), project SWFRG (Loera-

240 Sánchez, Studer, \& Kölliker, 2020). For single-plant libraries, variant calling and SNP-based 
241 diversity metrics were calculated as described above. Variant calling was performed

242 simultaneously on the BAM files of the sixteen single-plant libraries of each species. In the case

243 of pooled-plant libraries, amplicon-wise $\pi$ and SNP counts were calculated using the "Variance-

244 at-position.pl" script from PoPoolation v1.2.2 (Kofler et al., 2011) with a minimum covered

245 fraction of 0.5 , a pool size of 32 (i.e., the equivalent of 16 diploid plants), a minimum site quality

246 of 20 , a minimum coverage of 10 , and a minimum allele count of 5 . The PoPoolation analysis

247 was run individually for each BAM file of the pooled-plant libraries.

248 SNP haplotypes were reconstructed by concatenating each SNP position from each sequencing

249 read for both single- and pooled-plant samples. For this, BAM files containing the read mappings

250 to the amplicon pseudo-reference assemblies were parsed to extract reads and SNP positions

251 using a custom Python script. The SNP positions came from the mappings of the single-plant

252 libraries. Determining the final haplotype-based genotype required filtering out spurious

253 haplotypes with low read counts, which likely are due to PCR or sequencing errors. Briefly, for

254 each amplicon, the haplotype with the maximum read count was determined. Such maximum

255 read count was used to normalize read counts for the rest of the haplotypes. Normalized

256 haplotype allele counts thus ranged from zero to one. Only haplotypes with normalized counts

257 higher than a specific cutoff value ( 0.9 for diploids, 0.3 for tetraploids and 0.2 for pools) were

258 retained. For single-species samples, haplotypes were further visually inspected, and the following

259 was corrected: a) haplotypes with missing values were removed, b) haplotypes with tri- or tetra-

260 allelic SNPs were removed and c) co-occurring haplotypes with only one different SNP allele call

261 were consolidated into one haplotype. No visual inspection was conducted for pooled-plant

262 samples, but only haplotypes that were present in single-species samples were retained. 
263 Haplotype-based multi-locus genotypes, pairwise Prevosti's distances, allele count and expected 264 heterozigosities were calculated using the 'poppr' R package (Kamvar, Tabima, \& Gr $\square$ unwald, 265 2014).

266 To calculate normalized $k$-mer richness, reads were extracted from de-replicated BAM files using 267 SAMtools bam2fq, creating separate FASTQ files for each amplicon within each library. Each 268 FASTQ file was then sub-sampled to contain 100 reads using Seqtk v1.3 (Seqtk-1.3, 2018) . K269 mers of $k=25$ in each sub-sampled FASTQ file were determined with jellyfish (Marçais \& 270 Kingsford, 2011). The resulting $k$-mer dumps were filtered by discarding $k$-mers with $<25 \mathrm{x}$ 271 sequencing depth. Amplicon-specific NKR was calculated at the species level as described above.

272 Total diversity per species was calculated using exclusively single-plant libraries. Calculations were conducted as described in the discovery phase. The count of SNP-based haplotypes (H.Ac) for all amplicons was also calculated for each species. Genetic diversity metrics for each sample were compared to the diversity of the DNA barcode $r b c L a$, which is known to have a very low within276 species diversity.

SSR and multi-locus analysis

278 Single-plant DNA samples were also used as templates for SSR analyses. In total, eight species279 specific SSR primer pairs were used. SSR primers and PCR conditions are described in 280 Supplementary Table 5. Genotype tables were analyzed using the R package 'poppr' (Kamvar et 281 al., 2014). Summary statistics per locus are shown in Supplementary Table 6.

282 The five amplicons with the highest coverage per species were selected for multi-locus analysis 283 (AMP469 was excluded for T. repens as it is potentially duplicated in this species; Table 8, column 284 3). Only amplicons from single-plant libraries were analyzed. Accordingly, the five most diverse 285 SSRs per species were selected for multi-locus analysis (again, four for T. repens; Table 8, column $2864)$. 
287 Multi-locus genotypes were produced for SSRs (from now on "SSR-MLGs") and for amplicon-

288 based haplotypes (from now on "HAP-MLGs") using the R package 'poppr' (Kamvar et al.,

289 2014). Pairwise Prevosti's distance was calculated for SSR- and HAP-MLGs using the R package

290 'poppr' (Kamvar et al., 2014). Plants with >5\% missing loci, either amplicons or SSRs, were

291 removed from this analysis.

292 In addition, multi-locus $k$-mer distances were calculated using sourmash (Titus Brown \& Irber,

293 2016). For this, the normalized FASTQ files described above were merged into multi-amplicon

294 FASTQ files according to library name. Only FASTQ files corresponding to single-plant libraries

295 and to the amplicons selected for amplicon-MLGs were merged. Multi-amplicon FASTQ files

296 were used to calculate $k$-mer signatures using sourmash sketch (Titus Brown \& Irber, 2016).

297 Finally, pairwise similarities among samples of the same species were calculated using sourmash

298 compare (Titus Brown \& Irber, 2016). Distances were calculated from similarities by applying the

299 formula: $d=1-s$, were $d$ is distance and $s$ is simalirity. The ratios among the three kinds of

300 distances were calculated for each pair of plants and summarized in Table 8. 


\section{$301 \quad$ Results}

302 Discovery phase

303 Target loci selection and bait design

304 A total of 611 loci (265 ULEs and 346 orthologous genes) were selected, for which 12,253, 100-

305 nucleotide long baits were designed (5,526 targeting ULEs and 6,727 orthologous genes). In total,

306602 loci mapped to a gene model in any of the three reference genomes used as controls ( $A$.

307 thatiana, B. distachyon and M. truncatula). Of those loci, 365 were present in all three reference

308 genomes, 142 were present in only in A. thaliana and B. distachyon, 31 in A. thaliana and $M$.

309 truncatula, 31 in B. distachyon and M. truncatula, ten only in A. thaliana, ten in B. distachyon and

310 thirteen in M. truncatula (Supplementary Table 2 and Supplementary Figure 1).

311 In any of the three reference genomes, a total of 85 genes contained two or more target loci and,

312 conversely, 23 loci mapped to two or more genes (Supplementary Table 3).

313 Sequence capture and sequencing

314 Around 14.5 million raw read pairs were obtained after sequence capture, 10.3 million reads for

315 grasses and 4.2 million for legumes (Table 2). At the species level, total raw output ranged from

316512,233 read pairs for $T$. flavescens to $\sim 1.5$ million read pairs for $L$ corniculatus. The N50 of

317 pseudo-RAs generated from the pooled-plant samples ranged from 590 bp (T. pratense) to 4,905

318 bp (A. pratensis).

319 Approximately two million read pairs were successfully quality-controlled, merged and mapped to

320 a target locus. This constitutes a total capture efficiency of $13.45 \%$ of the total raw reads. Within

321 grasses and legumes, capture efficiencies were 4.78\% (495,858 quality-controlled, merged reads)

322 and $35 \%$ ( $\sim 1.5$ million quality-controlled, merged reads), respectively. At the species level,

323 capture efficiency varied from $1.58 \%$ for $A$. elatius $(18,735$ quality-controlled, merged reads) to 
$32455.82 \%$ for T. pratense (537,289 quality-controlled, merged reads). Read duplication in quality-

325 controlled, merged reads ranged from $12.75 \%$ in F. mbra to $38.59 \%$ in T. pratense.

326 For further analysis, only quality-controlled loci (QCL: loci with five or more quality-controlled,

327 merged reads within a library) and quality-controlled libraries (QC-libs: libraries with >100 QCL

328 and $>1,000$ quality-controlled, merged reads) were considered. Read counts were always

329 calculated as quality-controlled, merged reads. In total, 60 QC-libs with a median of 438 QCL per

330 library (interquartile range, IQR = 355-532; Table 2) were obtained. Twenty-three loci were not

331 captured in any library. Each QCL had a median of 37 reads (IQR $=17-84$ ). For grasses, there

332 were 37 QC-libs with a median of 377 QCL per library (IQR = 313 - 410); median reads per

333 grass QCL was 21, (IQR = 12 - 36). For legumes, there were 23 QC-libs with a median of 548

334 QCL (IQR = 520 - 552); median reads per legume QCL was 84 (IQR = 48 - 136). At the species

335 level, QC-lib varied from one for T. flavecens to a maximum of five obtained for C. cristatus, $D$.

336 glomerata, L. comiculatus, O. viciifolia and T. pratense. Median read count per QCL varied from 13 in

337 L. perenne (IQR $=9-20)$ to 149 in T. pratense (IQR = $97-227)$. Median QCL count varied from

338213 for $A$. elatius (IQR $=190-233)$ to 579 for T. repens $(\mathrm{IQR}=574-584)$.

\section{Genetic diversity estimations}

340 Diversity statistics were calculated for each locus within each species. Overall, normalized $k$-mer

341 richness (NKR) values ranged from zero (locus ortho-1479 in T. flavescens) to 13.04 (locus ortho-

342147 in $T$. repens) with a median of 1.06 (IQR $=0.82-1.35)$. SNP count ranged from one (326

343 locus-species pairs) to 86 (loci ortho-1288/uce-11005138 in Phleum pratense) with a median of 9

344 (IQR = 4 - 15). SNP densities ranged from 1 (36 locus-species pairs) to 99.83 (ortho-1178 in $T$.

345 pratense) with a median of $10.79(\mathrm{IQR}=5-19.27)$. Nucleotide diversity $(\pi)$ ranged from $2.5 \times 10^{-4}$

346 (ortho-1106 and uce-12001183 in $M$. sativa) to $5.17 \times 10^{-2}$ (ortho-1186/uce-11003333 in $F$.

347 pratensis $)$ with a median of $4.99 \times 10^{-3}\left(\mathrm{IQR}=2.33 \times 10^{-3}-9.08 \times 10^{-3}\right)$. 
348 In general, NKR and $\pi$ showed a very low correlation $\left(r^{2}=0.08\right.$, Fig. 1a). At the species-level, $r^{2}$

349 values ranged from 0.1 for $P$. pratensis to 0.47 in $L$. multiflorum, while for most species $r^{2} \geq 0.20$

350 (Fig. 1b).

351 Locus selection was based on such median diversity values across species, as well as in species

352 count (i.e., the number of species were a locus was found) and its suitability for multispecies

353 primer design. Across species, median NKR values ranged from 0.45 (ortho-350) to 8.78 (ortho-

354 106). Median SNP count ranged from 1 SNP (ortho-1474 and ortho-1498) to 43 SNPs (uce-

355 11005074). Median SNP densities ranged from 1.01 (ortho-1474) to 49.7 (ortho-1198). Median $\pi$

356 ranged from $5.3 \times 10^{-4}$ (ortho-1498) to $2.1 \times 10^{-2}$ (uce-11005074). Median diversity metrics across

357 species for the selected loci are shown in Table 4. A Wilcoxon test showed significant differences

358 (P-value $<0.001)$ between selected and non-selected loci based on the median of medians of

359 NKR, SNP count, SNP density per kbp and $\pi$ (Fig. 1c). 
360

361

362

363

364

365

366

367

368

369

370

371

372

373

374

375

376

377

378

380

381

382

383

\section{$V$ alidation phase}

\section{Multispecies amplicon sequencing and genetic diversity analysis}

Amplicon sequencing in test populations ( $\mathrm{n}=16$ ) of $D$. glomerata, F. pratensis, L. perenne, T. pratense and T. repens produced 12.4 million raw read pairs of which 4.3 million reads $(34.81 \%)$ were successfully quality-controlled and merged. Raw output was 6.5 million reads for grasses, with 1.8 million quality-controlled, merged reads $(26.77 \%$ of raw output for this plant family). For legumes, raw output was $\sim 5.9$ million reads, with 2.5 million quality controlled, merged reads (43.72\%). At the species level, raw output was 1.99, 2.25, 2.30, 3.03 and 2.86 million reads for $D$. glomerata, F. pratensis, L. perenne, T. pratense and T. repens, respectively. In turn, quality-controlled, merged read counts were 0.57 (28.79\% of raw output for this plant species), $0.64(28.34 \%), 0.54$ (23.49\%), $1.51(49.67 \%)$ and $1.07(37.42 \%)$ million reads for D. glomerata, F. pratensis, L. perenne, T. pratense and T. repens, respectively. Duplicate rate was always $>90 \%$ in all groups (total, plant families and species).

At the locus level, quality-controlled amplicons (i.e., amplicons with $\geq 5$ mapped reads) reported a median of $>1,000$ reads per library. Median count of quality-controlled amplicons was eight in $L$. perenne and T. pratense libraries, and seven in the rest of the species. The DNA barcode $p s b K-p s b I$ could not be recovered from legume samples, so it was excluded from analysis. Furthermore, amplicon AMP3794 had zero coverage in T. repens libraries. In addition, amplicon AMP3437 had zero coverage in D. glomerata and F. pratense libraries. The latter case is because AMP3437 targets the same locus as AMP3425 so, from here on, AMP3437 reads were treated as AMP3425 reads.

In single-plant libraries and excluding DNA barcode $r b c \mathrm{a} a$, total NKR values ranged from 1 ( $F$. pratensis) to 2.05 (T. repens). Total SNP counts ranged from 27 (F. pratensis) to 62 (D. glomerata). Total $\pi$ ranged from $5.19 \times 10^{-3}$ to $1.32 \times 10^{-2}$. Median SNP-based haplotype counts ranged from 19 (F. pratensis) to 43 (L. perenne). 
384 In single-plant libraries and excluding DNA barcode $r b c t a$, NKR ranged from zero (AMP3625 in

385 F. pratensis) to 2.94 (AMP3799 in D. glomerata) with a median of 1.53 (IQR $=1.02-1.97$ ). SNP

386 count ranged from zero (nine species-locus pairs) to 28 (AMP3799 in D. glomerata) with a median

387 of 3 (IQR = 0-11). SNP densities ranged from zero (nine species-locus pairs) to $50.36 \mathrm{SNPs} / \mathrm{kbp}$

388 (AMP3799 in D. glomerata) with a median of $6.91(\mathrm{IQR}=0-20.14) . \pi$ ranged from zero (nine 389 species-locus pairs) to $3.83 \times 10^{-2}$ (AMP3799 in D. glomerata) with a median of $4.62 \times 10^{-3}$ (IQR $=0$ -

$3901.52 \times 10^{-2}$ ). SNP-based haplotype allele count (H.Ac) ranged from one (11 cases) to 25

391 (AMP3799 in D. glomerata) with a median of 3 (IQR $=1-7$ ).

392 In pooled-plant libraries, NKR values ranged from zero (AMP3625 in F. pratensis pool 2) to 3.62

393 (AMP3799 in D. glomerata pool 1) with a median of 1.62 (IQR = $1.01-2.21)$. SNP counts ranged

394 from zero (38 species-locus pairs) to 54 (AMP3799 in D. glomerata pools 1) with a median of 2

395 (IQR = 0 - 19). SNP density ranged from zero (38 species-locus pairs) to $101.82 \mathrm{SNPs} / \mathrm{kbp}$

396 (AMP3799 in D. glomerata pool 3) with a median of 3.77 (IQR $=0-35.83$ ). $\pi$ ranged from 0 (38

397 species-locus pairs) to $6.68 \times 10^{-2}$ (AMP3425 in L perenne pool 1) with a median of $3.13 \times 10^{-3}$ (IQR

$398=0-1.51 \times 10^{-2}$ ). H.Ac ranged from one (41 species-locus pairs) to 22 (AMP3799 in D. glomerata pool 1) with a median of $2(\mathrm{IQR}=1-5)$.

400 The concordance between diversity metrics calculated for each species-amplicon pair from 401 single-plant libraries and pooled-plant libraries was good. Overall, the coefficients of variation of 402 all diversity metrics for each amplicon and species had a median of $13.38 \%$ (IQR $=3.35 \%-$ $40327.58 \%$ ). The coefficient of variation (CV) of NKR estimates ranged from $0.11 \%$ (AMP3532 in 404 F. pratensis and $r b c L a$ in T. pratense) to $86.67 \%$ (AMP3625 in F. pratensis) with a median of $8.1 \%$ $405(\mathrm{IQR}=2.43 \%-16.68 \%)$. The range of the CV of SNP counts ranged from $0 \%$ (16 species406 amplicon pairs) to $88.79 \%$ (AMP3425 in $L$. perenne) with a median of $12.87 \%$ (IQR $=0 \%$ $40727.75 \%$ ). In the case of SNP densities, CV ranged from $0 \%$ (12 species-amplicon pairs) to 
$90.60 \%$ (AMP3425 in $L$ perenne) with a median of $10.10 \%$ (IQR $=0 \%-28.65 \%$ ). The CV of $\pi$

409 estimates ranged from $0 \%$ (twelve species-amplicon pairs) to $114.47 \%$ (AMP3425 in $L$. perenne)

410 with a median of $15.43 \%$ (IQR $=0 \%-26.06 \%$ ). Finally, the CV of SNP-based haplotype counts

411 ranged from 0\% (22 species-amplicon pairs) to 119.02\% (AMP3425 in $L$ - perenne) with a median

412 of $0 \%(\mathrm{IQR}=0 \%-23.14 \%)$.

413 At the amplicon level and across species, median CV of NKR estimates ranged from $1.36 \%$

414 (AMP3532) to 56.70\% (AMP3625). For SNP counts, median CV ranged from 0\% (AMP615,

415 AMP3411, AMP3941 and AMP3794) to 88.79\% (AMP3425). For SNP densities, median CV

416 ranged from $0 \%$ ( $r b c \mathrm{a} a$ and AMP3941) to $90.60 \%$ (AMP3425). For $\pi$ estimates, median CV

417 ranged from $0 \%$ ( $r b c \mathrm{~L} a$ and AMP3941) to 114.47\% (AMP3425). For SNP-based haplotype

418 counts, median CV ranged from 0\% ( $r b c$ a, AMP3941, AMP615, AMP3411, AMP3625,

419 AMP3794 and AMP3425) to 28.57\% (AMP3376).

420 Across species, the amplicons were significantly more diverse than the DNA barcode $r b c I a$ for at

421 least one diversity metric (Fig. 3a). However, amplicon AMP469 in T. repens produced SNP-based

422 haplotypes that do not match the ploidy of this species (i.e., tetraploid). This amplicon was not

423 considered for overall diversity calculations (Table 6) nor for comparisons with SSR-based

424 diversity metrics.

425 Sequence-based diversity metrics showed a weak positive correlation to each other $\left(0.25 \leq r^{2} \leq\right.$

4260.32 , Figure $2 \mathrm{~b}$, black). Excluding amplicons without SNP calls marginally improved correlations

$427\left(0.3 \leq r^{2} \leq 0.45\right.$, Figure $2 b$, blue). Excluding amplicons without SNP calls and considering only

428 single-plant libraries resulted in considerably higher correlations $\left(0.51 \leq r^{2} \leq 0.69\right.$, Figure $2 b$, red). 
$430 K$-mer based distances had an overall median of $0.29(\mathrm{IQR}=0.21-0.38)$. At the species level, median k-mer-based distances were $0.34(\mathrm{IQR}=0.28-0.39)$ for D. glomerata, $0.20(\mathrm{IQR}=0.15$ -

$0.43)$ for F. pratensis, $0.31(\mathrm{IQR}=0.22-0.40)$ for $L$. perenne, $0.28(\mathrm{IQR}=0.19-0.39)$ for $T$.

pratense, and $0.24(\mathrm{IQR}=0.21-0.30)$.

434 Prevosti's pairwise distances based on HAP-MLGs had an overall median of 0.25 (IQR $=0.12$ 435 0.32). At the species level, median distances based on HAP-MLGs were 0.28 (IQR $=0.25-0.32$ )

436 for D. glomerata, 0.25 (IQR $=0.16$ - 0.30) for $F$. pratensis, 0.28 (IQR $=0.22-0.32)$ for $L$. perenne, $4370.45(\mathrm{IQR}=0.40-0.50)$ for T. pratense and $0.09(0.06-0.12)$ for T. repens.

438 Prevosti's pairwise distances based on SSR-MLGs had an overall median of $0.60(\mathrm{IQR}=0.50$ $4390.70)$. At the species level, median distances based on SSR-MLGs were 0.55 (IQR $=0.50-0.65$ )

440 for D. glomerata, $0.50(\mathrm{IQR}=0.40-0.60)$ for $F$. pratensis, $0.50(\mathrm{IQR}=0.40-0.55)$ for $L$. perenne, $0.90(\mathrm{IQR}=0.83-1.00)$ for T. pratense and $0.62(\mathrm{IQR}=0.56-0.69)$ for T. repens.

Distances based on SSR-MLGs were larger than both k-mer- and HAP-MLGs distances in all species (Fig. 4). In turn, k-mer-based distances were significantly larger than HAP-MLGs

444 distances in all species except F. pratensis (no significant differences between $k$-mer- and 445 haplotype-based distances, Fig. 4) and T. pratense (haplotype-based distances were larger than k446 mer based, Fig. 4).

447 In grasses, $k$-mer- and haplotype-based distances are very similar to each other (i.e., their ratio is 448 close to 1; Table 8), whereas in legumes, they are more dissimilar (i.e., their ratio deviates from 1; 449 Table 8). 


\section{Discussion}

451 We observed capture efficiencies (i.e., the proportion of the total sequencing read output that

452 maps on the target loci; Table 2) ranging from $1.58 \%$ to $55.82 \%$ per species, a range that is in line

453 with previous reports of multispecies sequence capture assays. For example, a study of 25 legume

454 species reported capture efficiencies ranging from $17 \%$ to $48 \%$ per species (Vatanparast et al.,

455 2018). Another study that analyzed 42 angiosperm species reported a wider range of capture

456 efficiencies per species: 5\% to $68.1 \%$ (Johnson et al., 2019). Grass and legume species had

457 different capture efficiencies, which is likely due to grasses having larger average genome sizes

458 (4,838.5 Mbp) than legumes (958.4 Mbp; Supplementary Table 7). As libraries with similar

459 concentrations were pooled together disregarding their species in each sequence capture reaction,

460 this could have resulted in a lower effective concentration of target loci for grass samples in the

461 discovery phase of this study. This is supported by a negative linear correlation observed between

462 genome size and capture efficiency $\left(r^{2}=0.58, P\right.$-value $\left.<0.001\right)$. On average and depending on the

463 species, $34.9 \%$ to $94.8 \%$ of the 611 target loci per species were recovered (Table 2). A similarly

464 high, species-dependent variability of captured loci has been observed in other multispecies

465 studies, from 54\% to $98 \%$ (Vatanparast et al., 2018) or even 2\% to 98\% (Johnson et al., 2019).

466 We expected our estimates of genetic diversity based on the sequence capture assay to be lower

467 than genome-wide genetic diversity estimates of forage crop species. This is because the large

468 proportion of coding sequences and ultra-conserved-like elements in our target loci, which were

469 included to increase the chances of finding multispecies priming sites around polymorphic

470 sequences. SNP density per species (Table 3) were lower than a genome-wide estimate in $I$.

471 multiflorum (31 SNPs/kbp; Knorst et al., 2019) and than an estimate based on genic regions in $L$.

472 perenne (35.6 SNPs/kbp; Ruttink et al., 2015). The SNP densities we found in our sequence 
473 capture data (Table 3) are closer to the $17 \mathrm{SNPs} / \mathrm{kbp}$ reported for a set of plastomes from a

474 comprehensive sampling of D. glomerata from western Europe (Hodkinson et al., 2019).

475 Three main criteria to choose candidate loci for the validation phase were followed: a) a high

476 NKR value, b) high SNP-based diversity (i.e., high $\pi$ and SNP density) and c) suitability for 477 multispecies primers with predicted amplicon sizes of $450 \pm 50 \mathrm{bp}$. Such an amplicon length was 478 chosen so the longest possible haplotypes could be generated after merging Illumina MiSeq reads.

479 NKR and SNP-based diversity measures are two complementary ways to assess diversity. NKR is 480 the count of unique $k$-mer from reads mapping to a locus divided by the locus length. Such 481 measure accounts for insertion/deletion (indel) variants that go undetected by standard SNP 482 calling. As a tool to detect intraspecific variation, $k$-mer richness (or $k$-mer count) analysis has 483 been applied in barley sequences to detect apparent heterozygous mappings, i.e., clusters of 484 divergent sequencing reads that map to genomic loci that are closely related (Pérez-Cantalapiedra 485 et al., 2018). $K$-mer richness analysis has also been applied to infer haplotype diversity in viral populations (Malhotra et al., 2013).

We observed a low to moderate positive correlation between NKR and $\pi$ per $\mathrm{kbp}$ when analyzing each species individually (Fig. 2b), but no significant correlation was present in ungrouped data (Fig. 2a). This is likely because the pseudo-reference assemblies underlying our SNP and $k$-mer analyses were generated on a species-by-species basis. In addition, insertion/deletions (i.e., indel)

491 size variability was high (Supplementary Figure 10), causing poor correlations SNP-based metrics 492 and NKR.

493 The sequence capture approach of the discovery phase of this work was developed as a steppingstone to find suitable loci for multispecies amplicon sequencing; however, it can per se be useful for other applications that justify its relatively high cost. The FORAGE-611 bait set could 
497 resolve phylogenetic relationships in closely related clades, such as the Festuca-Lolium complex

498 (Cheng et al., 2016), which comprises some of the most widely cultivated forage grasses in 499 temperate climates It can also be a helpful tool to study intergeneric hybridization and 500 allopolyploidization, a prevalent feature in grasses (Tkach et al., 2020).

501 In the validation phase of this study, we focused on eleven polymorphic loci and the DNA 502 barcode rbcta (Table 1), which were amplified and sequenced from single- and pooled-plant 503 samples of D. glomerata, F. pratensis, L. perenne, T. pratense and T. repens. We initially designed a set 504 of $>30$ candidate multispecies primer pairs targeting $>20$ loci, but we only picked those with high 505 efficiency in test PCRs (results not shown). More working multispecies primer pairs targeting 506 more loci could be generated by varying amplicon size and by further primer optimization.

507 However, this was out of the scope of this proof-of-concept study.

508 As expected from amplicon sequencing data, sequencing coverage per locus was very high 509 (>1,000x). Most of the selected amplicons were polymorphic, which made them useful for 510 genetic diversity assessment (Fig. $3 a$ and Table 5). In general, genetic diversity in single- and 511 pooled-plant libraries were mostly similar, with some variation due to the diversity metric and the 512 amplicon.

513 Among diversity metrics, NKR had the lowest variation due to sample pooling. This highlights

514 the robustness of alignment-free methods for genetic diversity studies. SNP-based metrics (i.e.,

515 SNP count, SNP density and $\pi$ ) had higher variations, likely due to the differences between the 516 SNP calling software. PoPoolation produced more SNP calls in the pooled-plant libraries 517 compared to the SNP calls of BCFtools mpileup in their matching single-plant libraries. This is to 518 be expected because PoPoolation false SNP discovery rates are known to increase with depth 519 (Raineri et al., 2012). In the case of SNP-based haplotypes, the low variation haplotype counts 
520 stems from the way such haplotypes were generated in pooled-samples (i.e., only the haplotypes

521 that were present in the single-plant libraries were considered).

522 Among amplicons, those with low variability due to sample pooling (median $\mathrm{CV}<10 \%$ ) were

523 rbcla, AMP3411, AMP615, AMP3941 and AMP3794, which are also among the least diverse

524 amplicons (Table 7). In the rest of the amplicons, the moderate levels of variability of diversity

525 metrics (median CV between $12.54 \%$ to $33.19 \%$ ) is also likely a result of differences in SNP

526 calling software. However, correlations among amplicon diversity metrics were better than those

527 observed in the discovery phase of this study (Fig. 3b). This is likely because indels in our

528 multispecies amplicons had less size variability than the 611 loci captured in the discovery phase

529 (Supplementary Figure 3), which in turn reduced the discrepancy between NKR and SNP-based

530 metrics.

531 The average genetic diversity per species (mean $\pi=8.94 \times 10^{-3}$; mean SNP density $=12.04$

532 SNPs/kbp; Table 6) was higher than the diversity reported in another targeted sequencing study,

533 which analyzed nine genes putatively involved in flowering (overall $\pi=7.90 \times 10^{-3}$; overall SNP

534 density $=7.87$ SNPs/kbp; Fiil et al., 2011). Such study was carried out using twenty $L$. perenne

535 genotypes from different European sources (i.e., the "Lolium Test Set" or LTS). In contrast, the

536 average genetic diversity per species (Table 6) was lower than those reported for the LTS in

537 eleven pathogen resistance genes $\left(\pi=3.14 \times 10^{-2}\right.$; total SNP density $\approx 100 \mathrm{SNPs} / \mathrm{kbp}$; Xing et al.,

538 2007), which are subject to constant selection pressure and co-evolving alongside pathogens and,

539 therefore, are highly variable. The moderate levels of genetic diversity of our multispecies

540 amplicons compared to resistance genes is likely because our target loci are not involved in a

541 common biological process under evolutionary pressure. Our target loci were picked based on

542 their median within-species diversity across multiple forage species and based on their suitability

543 for multispecies amplification. 
544 To benchmark the diversity estimations from multispecies amplicons, pairwise genetic distances

545 based on $k$-mers and SNPs were compared to pairwise distances produced with SSRs, a highly

546 polymorphic and multi-allelic marker system. Multispecies amplicons underestimated true

547 pairwise genetic distances by $\sim 50 \%$ (Fig. 4 and Table 7). This indicates that more multispecies

548 amplicons are needed to improve their discriminatory power. Theoretically, the discriminatory

549 power of a few tens of SSRs is equivalent to roughly a hundred neutral SNPs (Kalinowski, 2002).

550 However, empirical evidence from an outbreeding plant species shows that the number of SNPs

551 needed to approximate genome-wide diversity estimations is higher than theoretical predictions

552 (Fischer et al., 2017). No correlation was observed between multispecies amplicon sequencing-

553 and SSR-based pairwise distances (data not shown), indicating that multispecies amplicons and

554 SSRs interrogate different parts of each species' genome, which results in different genetic

555 distance estimations for any given pair of genotypes. This highlights the large diversity present in

556 the genomes of outbreeding forage species.

557 In conclusion, we showed that multispecies amplicon sequencing is useful for genetic diversity

558 assessments in outbreeding forage crop species. The approach uses inexpensive, unmodified

559 primers and other conventional PCR reagents. By designing multispecies primers, amplicons are

560 transferable across many species of the Poaceae and Fabaceae families, which can further

561 diminish the costs and labor needed for large-scale, multispecies assessments. Compared to other

562 approaches like GBS and RAD-seq, the output of amplicon sequencing is highly enriched in

563 informative data, making it more resource efficient. Furthermore, amplicon sequencing data

564 greatly simplify bioinformatic analyses (M. Sato et al., 2019). We also showed that genetic

565 diversity estimates based on amplicon sequencing of pooled-plant material are similar to those of

566 single-plant material, which further reduces costs per sample. Since PCR is a very robust and

567 versatile method, improvements to the genetic diversity estimates based on multispecies

568 amplicon can be achieved by increasing the number of amplicons analyzed, by varying amplicon 
569 sizes, by varying PCR conditions (e.g., multiplex PCR can reduce the processing time needed for

570 each amplicon; Veeckman et al., 2019), or by adapting sequencing approaches (e.g., pool

571 sequencing can be used to characterize larger plant populations).

572 The tools presented in this study provide the basis for cost-effective, multispecies genetic

573 diversity assessments in grassland plants. In our view, these results represent a promising starting

574 point for further improvements and adaptations. As awareness increases around the ecological

575 significance of plant genetic diversity and as widespread monitoring of genetic diversity gains

576 traction (Hoban et al., 2020; Laikre et al., 2020; Pärli et al., 2021), such multispecies approaches

577 can be valuable additions to the toolset of genetic diversity monitoring efforts.

\section{References}

579 Bankevich, A., Nurk, S., Antipov, D., Gurevich, A. A., Dvorkin, M., Kulikov, A. S., ... Pevzner, 580 P. A. (2012). SPAdes: A New Genome Assembly Algorithm and Its Applications to Single581 Cell Sequencing. Journal of Computational Biology, 19(5), 455-477. doi: 10.1089/cmb.2012.0021

582 Bengtsson, J., Bullock, J. M., Egoh, B., Everson, C., Everson, T., O’Connor, T., ... Lindborg, R. 583 (2019). Grasslands-more important for ecosystem services than you might think. Ecosphere, $584 \quad$ 10(2), e02582. doi: 10.1002/ecs2.2582

585 Broad Institute. (2019). Picard Toolkit, Github Repository. Retrieved from 586 http://broadinstitute.github.io/picard/

587 Bushnell, B. (2014). BBMap. Retrieved from https://sourceforge.net/projects/bbmap/

588 Bushnell, Brian, Rood, J., \& Singer, E. (2017). BBMerge - Accurate paired shotgun read merging $589 \quad$ via overlap. PLoS ONE, 12(10), 1-15. doi: 10.1371/journal.pone.0185056

590 Caldu-Primo, J. L., Mastretta-Yanes, A., Wegier, A., \& Piñero, D. (2017). Finding a needle in a 
591

592

593

594

595

596

597

598

599

600

601

602

603

604

605

606

607

608

609

610

611

612

613

haystack: Distinguishing Mexican maize landraces using a small number of SNPs. Frontiers in Genetics, 8(APR), 1-12. doi: 10.3389/fgene.2017.00045

Carroll, E. L., Bruford, M. W., DeWoody, J. A., Leroy, G., Strand, A., Waits, L., \& Wang, J. (2018). Genetic and genomic monitoring with minimally invasive sampling methods. Evolutionary Applications, 11(7), 1094-1119. doi: 10.1111/eva.12600

Chen, S., Zhou, Y., Chen, Y., \& Gu, J. (2018). Fastp: An ultra-fast all-in-one FASTQ preprocessor. Bioinformatics, 34(17), i884-i890. doi: 10.1093/bioinformatics/bty560

Cheng, Y., Zhou, K., Humphreys, M. W., Harper, J. A., Ma, X., Zhang, X., ... Huang, L. (2016). Phylogenetic Relationships in the Festuca-Lolium Complex (Loliinae; Poaceae): New Insights from Chloroplast Sequences. Frontiers in Ecology and Evolution, 4(July), 1-12. doi: $10.3389 /$ fevo. 2016.00089

Collins, R. P., Helgadóttir, Á., Frankow-Lindberg, B. E., Skøt, L., Jones, C., \& Skøt, K. P. (2012). Temporal changes in population genetic diversity and structure in red and white clover grown in three contrasting environments in northern Europe. Annals of Botany, 110(6), 1341-1350. doi: $10.1093 / \mathrm{aob} / \operatorname{mcs} 058$

Cuyeu, R., Rosso, B., Pagano, E., Soto, G., Fox, R., \& Ayub, N. D. (2013). Genetic diversity in a world germplasm collection of tall fescue. Genetics and Molecular Biology, 36(2), 237-242. doi: $10.1590 /$ S1415-47572013005000021

Danecek, P., Auton, A., Abecasis, G., Albers, C. A., Banks, E., DePristo, M. A., ... Durbin, R. (2011). The variant call format and VCFtools. Bioinformatics, 27(15), 2156-2158. doi: 10.1093/bioinformatics/btr330

De Vega, J. J., Ayling, S., Hegarty, M., Kudrna, D., Goicoechea, J. L., Ergon, Å., ... Skøt, L. (2015). Red clover (Trifolium pratense L.) draft genome provides a platform for trait 
615 Faircloth, B. C. (2016). PHYLUCE is a software package for the analysis of conserved genomic 616 loci. Bioinformatics, 32(5), 786-788. doi: 10.1093/bioinformatics/btv646

617 Fiil, A., Lenk, I., Petersen, K., Jensen, C. S., Nielsen, K. K., Schejbel, B., ... Lübberstedt, T. 618 (2011). Nucleotide diversity and linkage disequilibrium of nine genes with putative effects on flowering time in perennial ryegrass (Lolium perenne L.). Plant Science, 180(2), 228-237. doi:

Fischer, M. C., Rellstab, C., Leuzinger, M., Roumet, M., Gugerli, F., Shimizu, K. K., ... Widmer, 10.1016/j.plantsci.2010.08.015

Hadincová, V., Skálová, H., Münzbergová, Z., \& Fischer, M. (2020). Genotypic diversity and genotype identity of resident species drive community composition. Journal of Plant Ecology, 13(2), 224-232. doi: 10.1093/jpe/rtaa004

Harvey, M. G., Smith, B. T., Glenn, T. C., Faircloth, B. C., \& Brumfield, R. T. (2016). Sequence Systematic Biology, 65(5), 910-924. doi: 10.1093/sysbio/syw036 A., ... Laikre, L. (2020). Genetic diversity targets and indicators in the CBD post-2020 Global Biodiversity Framework must be improved. Biological Conservation, 248(July), 108654. 
638 Hodkinson, T. R., Perdereau, A., Klaas, M., Cormican, P., \& Barth, S. (2019). Genotyping by 639 Sequencing and Plastome Analysis Finds High Genetic Variability and Geographical 640 Structure in Dactylis glomerata L. in Northwest Europe Despite Lack of Ploidy Variation. 641 Agronomy, 9(7), 342. doi: 10.3390/agronomy9070342

642 Huber, R., \& Finger, R. (2020). A Meta-analysis of the Willingness to Pay for Cultural Services 643 from Grasslands in Europe. Journal of Agricultural Economics, 71(2), 357-383. doi: $10.1111 / 1477-9552.12361$

Hysom, D. A., Naraghi-Arani, P., Elsheikh, M., Carrillo, A. C., Williams, P. L., \& Gardner, S. N. (2012). Skip the Alignment: Degenerate, Multiplex Primer and Probe Design Using K-mer Matching Instead of Alignments. PLoS ONE, 7(4), e34560. doi: The grapevine genome sequence suggests ancestral hexaploidization in major angiosperm Wickett, N. J. (2019). A Universal Probe Set for Targeted Sequencing of 353 Nuclear Genes from Any Flowering Plant Designed Using k-Medoids Clustering. Systematic Biology, 68(4), distances? Heredity, 88(1), 62-65. doi: 10.1038/sj.hdy.6800009

Kamvar, Z. N., Tabima, J. F., \& Gr $\square$ unwald, N. J. (2014). Poppr: An R package for genetic 659 analysis of populations with clonal, partially clonal, and/or sexual reproduction. PeerJ, 
661 Kawahara, Y., de la Bastide, M., Hamilton, J. P., Kanamori, H., McCombie, W. R., Ouyang, S., ... 662 Matsumoto, T. (2013). Improvement of the Oryza sativa Nipponbare reference genome 663 using next generation sequence and optical map data. Rice, 6(1), 4. doi: 10.1186/1939-8433$664 \quad 6-4$

665

666

667

668

669

670

671

672

Knorst, V., Byrne, S., Yates, S., Asp, T., Widmer, F., Studer, B., \& Kölliker, R. (2019). Pooled DNA sequencing to identify SNPs associated with a major QTL for bacterial wilt resistance in Italian ryegrass (Lolium multiflorum Lam.). Theoretical and Applied Genetics, 132(4), 947-958. doi: $10.1007 / \mathrm{s} 00122-018-3250-\mathrm{z}$

Kofler, R., Orozco-terWengel, P., De Maio, N., Pandey, R. V., Nolte, V., Futschik, A., ... Schlötterer, C. (2011). PoPoolation: A Toolbox for Population Genetic Analysis of Next Generation Sequencing Data from Pooled Individuals. PLOS ONE, 6(1), e15925. doi: 10.1371/journal.pone.0015925

Kölliker, R., Boller, B., Majidi, M., Peter-Schmid, M. K. I., Bassin, S., \& Widmer, F. (2009). Characterization and Utilization of Genetic Resources for Improvement and Management of Grassland Species. In Molecular Breeding of Forage and Turf (pp. 55-70). New York, NY: Springer New York. doi: 10.1007/978-0-387-79144-9_5

Laikre, L., Hoban, S., Bruford, M. W., Segelbacher, G., Allendorf, F. W., Gajardo, G., ... Vernesi, C. (2020). Post-2020 goals overlook genetic diversity. Science, 367(6482), 1083.2-1085. doi: $10.1126 /$ science.abb2748

Lamesch, P., Berardini, T. Z., Li, D., Swarbreck, D., Wilks, C., Sasidharan, R., ... Huala, E. (2012). The Arabidopsis Information Resource (TAIR): improved gene annotation and new tools. Nucleic Acids Research, 40(D1), D1202-D1210. doi: 10.1093/nar/gkr1090 
683 Last, L., Widmer, F., Fjellstad, W., Stoyanova, S., \& Kölliker, R. (2013). Genetic diversity of 684 natural orchardgrass (Dactylis glomerata L.) populations in three regions in Europe. BMC 685 Genetics, 14(1), 102. doi: 10.1186/1471-2156-14-102

686

Li, H. (2011). A statistical framework for SNP calling, mutation discovery, association mapping and population genetical parameter estimation from sequencing data. Bioinformatics, 27(21), 2987-2993. doi: 10.1093/bioinformatics/btr509

Li, H., Handsaker, B., Wysoker, A., Fennell, T., Ruan, J., Homer, N., ... Durbin, R. (2009). The Sequence Alignment/Map format and SAMtools. Bioinformatics, 25(16), 2078-2079. doi: 10.1093 /bioinformatics/btp352

Li, L., Stoeckert Jr., C. J., \& Roos, D. S. (2003). OrthoMCL: Identification of Ortholog Groups for Eukaryotic Genomes. Genome Research, 13(9), 2178-2189. doi: 10.1101/gr.1224503

Liu, S., Feuerstein, U., Luesink, W., Schulze, S., Asp, T., Studer, B., ... Dehmer, K. J. (2018). DArT, SNP, and SSR analyses of genetic diversity in Lolium perenne L. using bulk sampling. BMC Genetics, 19(1), 1-13. doi: 10.1186/s12863-017-0589-0

Loera-Sánchez, M., Studer, B., \& Kölliker, R. (2019). DNA-Based Assessment of Genetic Diversity in Grassland Plant Species: Challenges, Approaches, and Applications. Agronomy, 9(12), 881. doi: 10.3390/agronomy9120881

Loera-Sánchez, M., Studer, B., \& Kölliker, R. (2020). DNA barcode $\operatorname{trnH}-p s b A$ is a promising candidate for efficient identification of forage legumes and grasses. BMC Research Notes, 13(1), 1-6. doi: 10.1186/s13104-020-4897-5

Luo, M. C., Gu, Y. Q., Puiu, D., Wang, H., Twardziok, S. O., Deal, K. R., ... Dvoák, J. (2017). Genome sequence of the progenitor of the wheat D genome Aegilops tauschii. Nature, 551(7681), 498-502. doi: 10.1038/nature24486 
706 Malhotra, R., Prabhakara, S., Poss, M., \& Acharya, R. (2013). Estimating Viral Haplotypes in a

707 Population Using k-mer Counting. In A. Ngom, E. Formenti, J.-K. Hao, X.-M. Zhao, \& T. van Laarhoven (Eds.), Pattern Recognition in Bioinformatics (pp. 265-276). Berlin, Heidelberg: Springer Berlin Heidelberg.

Malyshev, A. V., Arfin Khan, M. A. S., Beierkuhnlein, C., Steinbauer, M. J., Henry, H. A. L., 711 Jentsch, A., ... Kreyling, J. (2016). Plant responses to climatic extremes: within-species variation equals among-species variation. Global Change Biology, 22(1), 449-464. doi:

Marçais, G., \& Kingsford, C. (2011). A fast, lock-free approach for efficient parallel counting of occurrences of k-mers. Bioinformatics, 27(6), 764-770. doi: 10.1093/bioinformatics/btr011

Martin, M. (2011). Cutadapt removes adapter sequences from high-throughput sequencing reads. EMBnet. Journal, 17(1), 10-12.

Mayer, C., Sann, M., Donath, A., Meixner, M., Podsiadlowski, L., Peters, R. S., ... Niehuis, O. (2016). BaitFisher: A Software Package for Multispecies Target DNA Enrichment Probe Design. Molecular Biology and Evolution, 33(7), 1875-1886. doi: 10.1093/molbev/msw056

Meilhac, J., Durand, J.-L., Beguier, V., \& Litrico, I. (2019). Increasing the benefits of species diversity in multispecies temporary grasslands by increasing within-species diversity. Annals of Botany, 123(5), 891-900. doi: 10.1093/aob/mcy227 identify candidate genes regulating pod color. Genome Biology, 14(6), r53. doi: 10.1186/gb2013-14-6-r53

Pärli, R., Lieberherr, E., Holderegger, R., Gugerli, F., Widmer, A., \& Fischer, M. C. (2021). 
Developing a monitoring program of genetic diversity: what do stakeholders say? Conservation Genetics, (0123456789). doi: 10.1007/s10592-021-01379-6

Pérez-Cantalapiedra, C., Contreras-Moreira, B., Casas Cendoya, A. M., \& Igartua Arregui, E. (2018). Unmasking new intra-species diversity through K-mer count analysis. EUCARPIA Cereal Section/IWW2 Meetings (Polydome - Clermont-Ferrand, France). Retrieved from https://digital.csic.es/handle/10261/162254

Prieto, I., Violle, C., Barre, P., Durand, J.-L., Ghesquiere, M., \& Litrico, I. (2015). Complementary effects of species and genetic diversity on productivity and stability of sown grasslands. Nature Plants, 1(4), 15033. doi: 10.1038/nplants.2015.33

Quinlan, A. R., \& Hall, I. M. (2010). BEDTools: A flexible suite of utilities for comparing genomic features. Bioinformatics, 26(6), 841-842. doi: 10.1093/bioinformatics/btq033

Raineri, E., Ferretti, L., Esteve-Codina, A., Nevado, B., Heath, S., \& Pérez-Enciso, M. (2012). SNP calling by sequencing pooled samples. BMC Bioinformatics, 13(1), 239. doi: 10.1186/1471-2105-13-239

Ratnasingham, S., \& Hebert, P. D. N. (2007). BOLD: The Barcode of Life Data System (http://www.barcodinglife.org). Molecular Ecology Notes, 7(3), 355-364. doi: 10.1111/j.14718286.2007.01678.x

Reynolds, S. G. (2005). Chapter 1 Introduction. In J. M. Suttie, S. G. Reynolds, \& C. Batello (Eds.), Grasslands of the World. Food and Agriculture Organization of the United Nations. Retrieved from http://www.fao.org/3/y8344e00.htm

Ruttink, T., Haegeman, A., van Parijs, F., Van Glabeke, S., Muylle, H., Byrne, S., ... Roldán-Ruiz, I. (2015). Genetic Diversity in Candidate Genes for Developmental Traits and Cell Wall Characteristics in Perennial Ryegrass (Lolium perenne). In H. Budak \& G. Spangenberg (Eds.), 
752

753

754

755

756

757

758

759

760

761

762

763

Molecular Breeding of Forage and Turf (pp. 93-109). Cham: Springer International Publishing. doi: 10.1007/978-3-319-08714-6_9

Sato, M., Hosoya, S., Yoshikawa, S., Ohki, S., Kobayashi, Y., Itou, T., \& Kikuchi, K. (2019). A highly flexible and repeatable genotyping method for aquaculture studies based on target amplicon sequencing using next-generation sequencing technology. Scientific Reports, 9(1), 19. doi: 10.1038/s41598-019-43336-x

Sato, S., Nakamura, Y., Kaneko, T., Asamizu, E., Kato, T., Nakao, M., ... Tabata, S. (2008). Genome structure of the legume, Lotus japonicus. DNA Research, 15(4), 227-239. doi: $10.1093 /$ dnares/dsno08

Sato, S., Tabata, S., Hirakawa, H., Asamizu, E., Shirasawa, K., Isobe, S., ... Gianese, G. (2012). The tomato genome sequence provides insights into fleshy fruit evolution. Nature, 485(7400), 635-641. doi: 10.1038/nature11119

Schmutz, J., Cannon, S. B., Schlueter, J., Ma, J., Mitros, T., Nelson, W., ... Jackson, S. A. (2010). Genome sequence of the palaeopolyploid soybean. Nature, 463(7278), 178-183. doi: $10.1038 /$ nature 08670

Schmutz, J., McClean, P. E., Mamidi, S., Wu, G. A., Cannon, S. B., Grimwood, J., ... Jackson, S. A. (2014). A reference genome for common bean and genome-wide analysis of dual domestications. Nature Genetics, 46(7), 707-713. doi: 10.1038/ng.3008

Seqtk-1.3. (2018). Retrieved from https://github.com/lh3/seqtk

Tang, H., Krishnakumar, V., Bidwell, S., Rosen, B., Chan, A., Zhou, S., ... Town, C. D. (2014). An improved genome release (version Mt4.0) for the model legume Medicago truncatula. BMC Genomics, 15(1), 1-14. doi: 10.1186/1471-2164-15-312

774 Tkach, N., Schneider, J., Döring, E., Wölk, A., Hochbach, A., Nissen, J., .. Röser, M. (2020). 
775

776

777

778

779

780

781

782

783

Phylogenetic lineages and the role of hybridization as driving force of evolution in grass supertribe Poodae. TAXON, 69(2), 234-277. doi: 10.1002/tax.12204

Vatanparast, M., Powell, A., Doyle, J. J., \& Egan, A. N. (2018). Targeting legume loci: A comparison of three methods for target enrichment bait design in Leguminosae phylogenomics. Applications in Plant Sciences, 6(3), e1036. doi: 10.1002/aps3.1036

Veeckman, E., Van Glabeke, S., Haegeman, A., Muylle, H., Van Parijs, F. R. D., Byrne, S. L., ... Ruttink, T. (2019). Overcoming challenges in variant calling: Exploring sequence diversity in candidate genes for plant development in perennial ryegrass (Lolium perenne). DNA Research, 26(1), 1-12. doi: 10.1093/dnares/dsy033

Vogel, J. P., Garvin, D. F., Mockler, T. C., Schmutz, J., Rokhsar, D., Bevan, M. W., .. Baxter, I. (2010). Genome sequencing and analysis of the model grass Brachypodium distachyon. Nature, 463(7282), 763-768. doi: 10.1038/nature08747

Xing, Y., Frei, U., Schejbel, B., Asp, T., \& Lübberstedt, T. (2007). Nucleotide diversity and linkage disequilibrium in 11 expressed resistance candidate genes in Lolium perenne. BMC Plant Biology, 7, 1-12. doi: 10.1186/1471-2229-7-43

Zhao, Y., Liu, Z., \& Wu, J. (2020). Grassland ecosystem services: a systematic review of research advances and future directions. Landscape Ecology, 35(4), 793-814. doi: 10.1007/s10980-02000980-3 


\section{Authors' contributions}

795 R.K. and B.S. conceived the study and provided insights on experimental design and data

796 analysis. M.L.-S. did the laboratory and bioinformatic analyses. All authors read and approved the

797 final manuscript.

\section{Acknowledgements}

799 We thank Franco Widmer and Christoph Grieder from Agroscope for giving us access to

800 greenhouses and laboratory space. Steven Yates (ETH Zurich) provided the list of orthologous, 801 single-copy genes used for bait design. Thanks to Damian Käch (ETH Zurich), who performed 802 the SSR genotyping of this study as part of his Bachelor thesis. Library preparations and 803 sequencing were performed at the Genetic Diversity Center of ETH Zurich. We thank M.

804 Hardegger and C. Kägi (Swiss Federal Office of Agriculture) for their valuable advice regarding 805 the design of the study. This work was funded by the Swiss Federal Office of Agriculture.

\section{Tables and figures}

807 


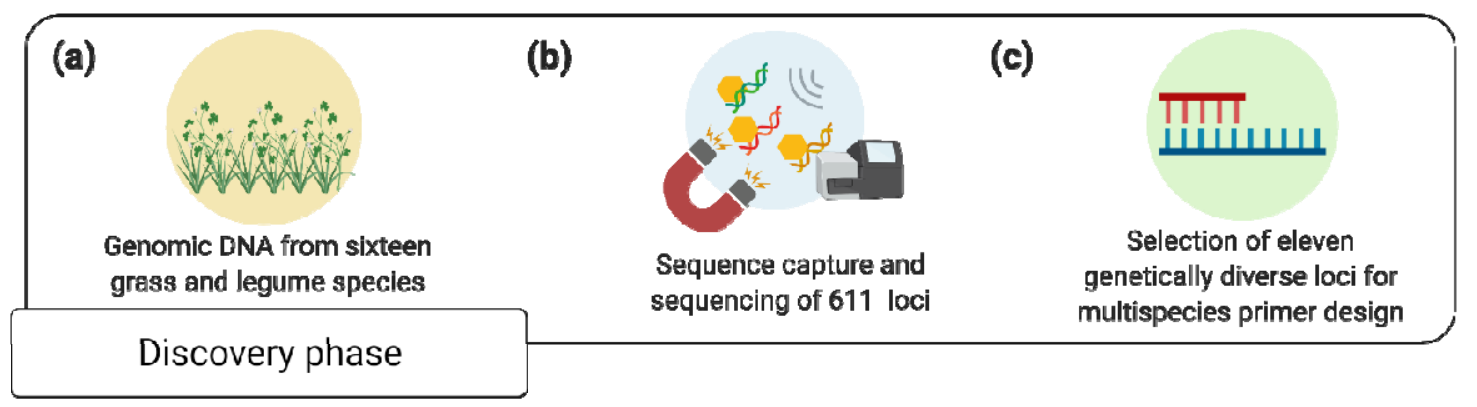

(d)

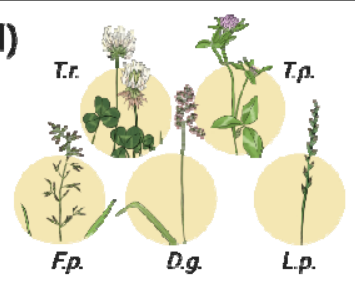

Genomic DNA from five grass and legurne species (e)

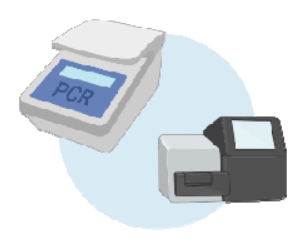

Amplicon sequencing, including barcode rbcla as a control (f)

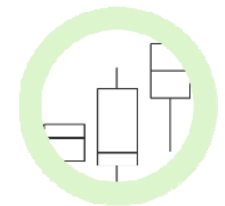

Comparison of multi-locus diversity of amplicons and SSRs

\section{Validation phase}

809 Figure 1 Graphical summary of the steps leading to the identification of multispecies 810 amplicons that were used for genetic diversity assessments. (a) Genomic DNA was 811 extracted from 16 grass and legume species (five plants from different cultivars per species). Each 812 species was represented by five single-plant samples and one pooled-plant sample. The pooled 813 plant sample of each species contained the same five plants used for single-plant samples. (b) 814 Illumina dual-indexed libraries were prepared ( $\sim 50 \mathrm{bp}$ fragment size) and sequenced. Libraries 815 were then used as targets for sequence capture. The target loci were 611 single-copy, orthologous 816 genes and ultra-conserved like elements. (c) Eleven loci were selected for multispecies primer 817 design, aiming for amplicons of $\sim 500 \mathrm{bp}$. (d) Genomic DNA was extracted from sixteen plants 818 from five grass and legume species (16 plants from three cultivars per species). Each species was 819 represented by sixteen single-plant DNA samples and three pooled-plant DNA samples. In each 820 species, pooled-plant samples included the same plants used for the single-plant samples. (e) 821 Multispecies amplicons were amplified all samples, including the DNA barcode $r b c \mathrm{a}$ as a 822 reference for a low-diversity locus. Amplicons were pooled equimolarly and dual-indexed libraries 823 and sequenced on the Illumina MiSeq platform. Diversity estimations took place as described 824 above. (f) The sixteen single-plant DNA samples were genotyped using eigth SSRs. The resulting 825 SSR multi-locus genotypes were compared to amplicon sequencing-based multi-locus genotypes, 826 in terms of their multi-locus genotype count and pairwise distances. Figure created with 827 BioRender.com. Art by Danira León https://www.behance.net/leondanira1c28. 
828 Table 1 Loci selected for amplicon sequencing and their corresponding multispecies

829 primer pairs sequences.

\begin{tabular}{|c|c|c|c|c|c|}
\hline Locus & $\begin{array}{c}\text { Corresponding } \\
\text { Arabidopsis thaliana } \\
\text { gene }\end{array}$ & Primer sequences (5' to 3') & $\begin{array}{l}\mathrm{Tm} \\
\left({ }^{\circ} \mathrm{C}\right)\end{array}$ & $\begin{array}{c}\text { Plant } \\
\text { family }\end{array}$ & $\begin{array}{l}\text { Amplicon } \\
\text { name }\end{array}$ \\
\hline O-1262 & AT3G07080 & $\begin{array}{l}\text { F: AAAGATTTGGATAGTAAAGCATGGA } \\
\text { R: TCCARCGYCCTTTYKCATCC }\end{array}$ & 60 & F & AMP469 \\
\hline U-11004576 & AT5G47010 & $\begin{array}{l}\text { F: AAGCTTGARGCTGAYTATGA } \\
\text { R: TGCATACCACATGACCMACT }\end{array}$ & 58 & F & AMP615 \\
\hline O-1520 & AT2G42900 & $\begin{array}{l}\text { F: ACACAAGCTCCTTTGTKGAA } \\
\text { R: ATACGATCATGCCACGTGTC }\end{array}$ & 58 & F & AMP735 \\
\hline O-165 & AT1G50480 & $\begin{array}{l}\text { F: GCTGATATGCGAGAGAGGCTAG } \\
\text { R: TAACATTCGCACCATAAGCT }\end{array}$ & 58 & F & AMP3411 \\
\hline O-1390 & AT1G08460 & $\begin{array}{l}\text { F: GGCACWTTCYTGAACCCTGG } \\
\text { R: GAATAACTTACTGCACTYGAGTC }\end{array}$ & 58 & F & AMP3711 \\
\hline U-11001396 & AT5G67170 & $\begin{array}{l}\text { F: GGGAGCATTATAAYAGTGTGCG } \\
\text { R: CTTCTGYWCCTTGTTCWGCT }\end{array}$ & 58 & F & AMP3794 \\
\hline O-1211 & AT5G61540 & $\begin{array}{l}\text { F: GTRGCACCATTGGTTGATGTG } \\
\text { R: GAARTHGGAGCTGTKGSTGC }\end{array}$ & 58 & FP & AMP3941 \\
\hline O-1347 & AT2G44020 & $\begin{array}{l}\text { F: GCGTGACATTGGTCCCATGG } \\
\text { R: GATCCTBGACTCCAAGCTGTA }\end{array}$ & 58 & $\mathrm{P}$ & AMP3376 \\
\hline $\begin{array}{l}\text { U-11004158/ } \\
\text { O-1519 }\end{array}$ & AT1G70570 & $\begin{array}{l}\text { F: GCTGGCATGACAATAAGAGC } \\
\text { R: WGTRCTCCTRAARAAGCGTGT }\end{array}$ & 58 & $\mathrm{P}$ & AMP3425 \\
\hline $\begin{array}{l}\text { U-11004158/ } \\
\text { O-1519 }\end{array}$ & AT1G70570 & $\begin{array}{l}\text { F: GCTGGCATGACAATAAGAGC } \\
\text { R: AAAAGCGTGTATTCCCATCATA }\end{array}$ & 60 & P & AMP3437 \\
\hline O-1288 & AT5G38460 & $\begin{array}{l}\text { F: GGAAGAGATTGTTTGCAATAAAGCC } \\
\text { R: CATTATCAGTGCATCAAAGAGGA }\end{array}$ & 58 & $\mathrm{P}$ & AMP3532 \\
\hline O-165 & AT1G50480 & $\begin{array}{l}\text { F: GGAGGTGGCTACAGTCAAGT } \\
\text { R: GTGGGATGAATAGCATCTTTCATT }\end{array}$ & 58 & P & AMP3625 \\
\hline O-1318 & AT5G04050 & $\begin{array}{l}\text { F: GGGAGGAGGTGCACAAGAAG } \\
\text { R: ACAACCGRTGCCARTAACGG }\end{array}$ & 58 & $\mathrm{P}$ & AMP3799 \\
\hline$r b c L a$ & $r b c L a$ & $\begin{array}{c}\text { F: ATGTCACCACAAACAGAGACT AAAGC } \\
\text { R: GTAAAATCAAGTCCACCRCG }\end{array}$ & 55 & FP & $r b c L a$ \\
\hline
\end{tabular}

830 †Plant family abbreviations: F = Fabaceae (legumes); P = Poaceae (grasses). 

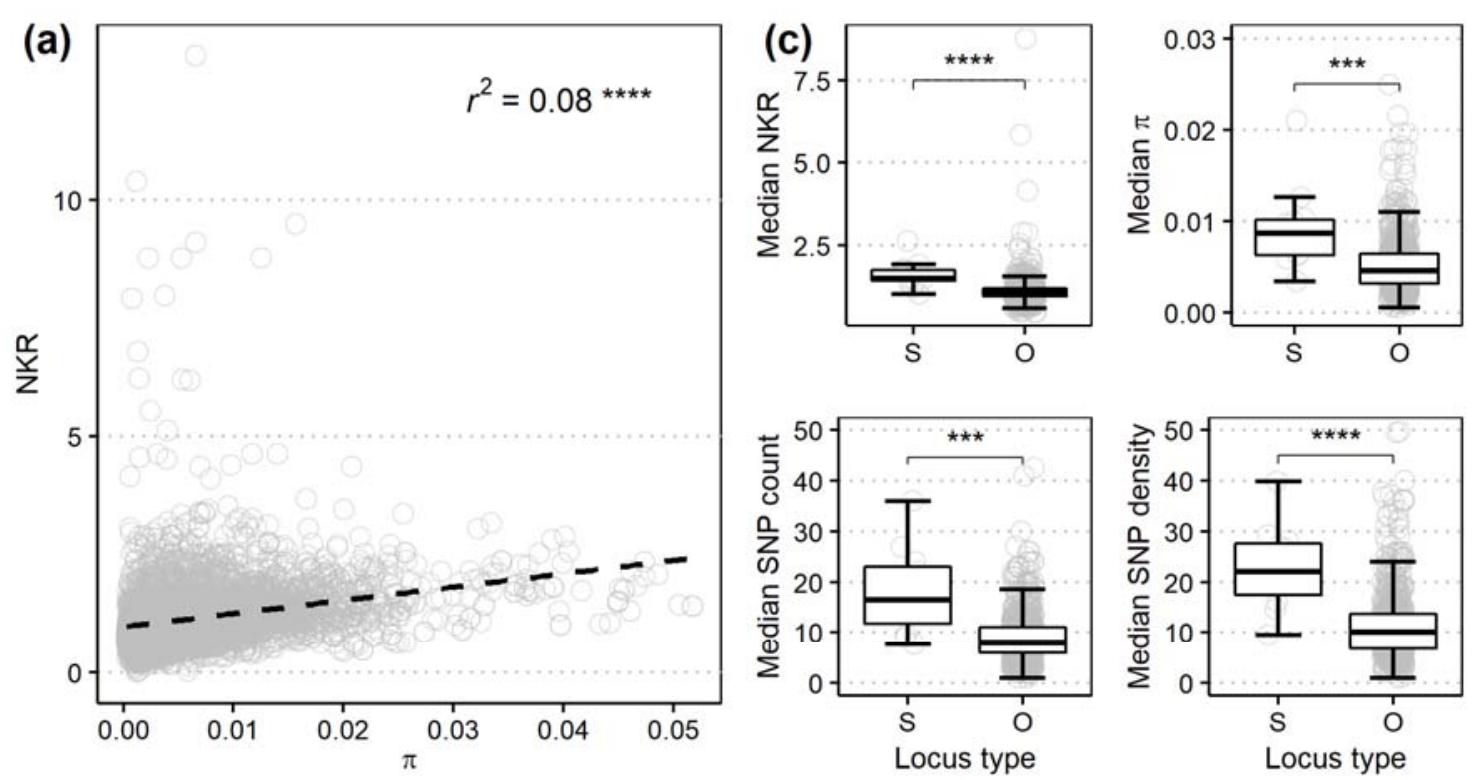

(b) Alopecurus pratensis
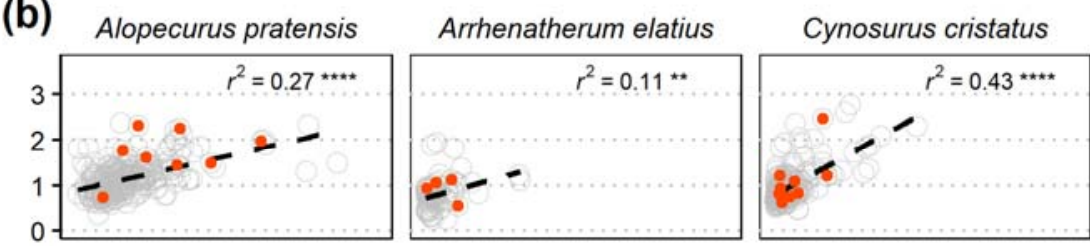

Dactylis glomerata
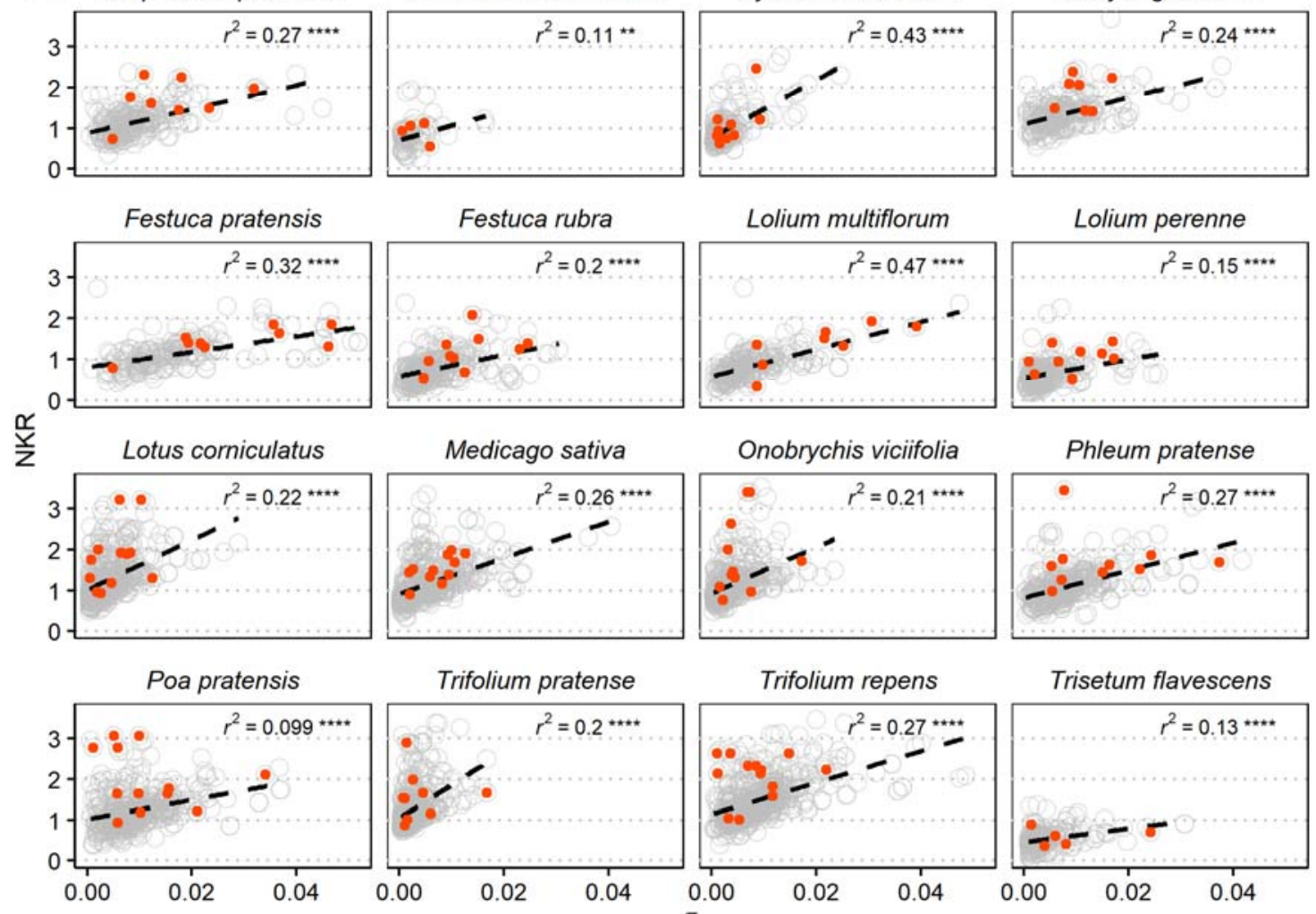

Lolium perenne

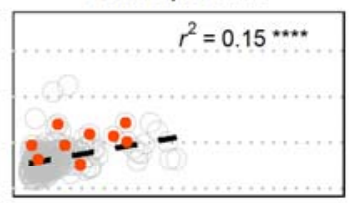

Phleum pratense
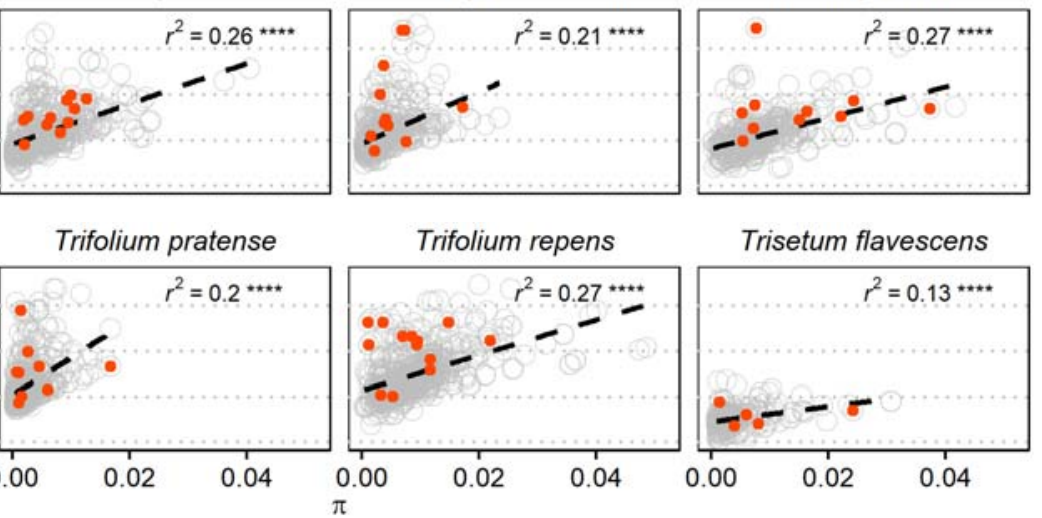

831

832 Figure 2 Diversity statistics of the captured loci. (a) Nucleotide diversity per kbp ( $\pi$ ) vs normalized $k$-mer richness (NKR) for all captured loci in the 60 quality-controlled libraries. Linear regression shown in black dotted line. Regression $r^{2}$ value and P-value significance level shown in upper right corner. (b) $\pi$ per kbp vs NKR for all captured loci divided by species. Red 
836 dots indicate selected loci. Linear regression shown in black dotted line. Regression $r^{2}$ value and $837 P$-value significance level shown in bottom right. (c) A Wilcoxon test shows that median 838 diversity metrics across the sixteen species are higher in selected loci ("S" label, $\mathrm{n}=10$ ) than in 839 other captured loci ("O" label, $\mathrm{n}=578$ ). Significance levels for $P$-values: $0.0001=* * * *, 0.001=$ $840 * * *, 0.01=* *, 0.05=*$, ns $=$ non-significant. The upper and bottom ends of boxplots indicate 841 the $3^{\text {rd }}$ and $1^{\text {st }}$ quartiles, respectively. The black line inside of boxplots indicates the median value. 842 The upper and lower whiskers in boxplots indicate the maximum $\left(3^{\text {rd }}\right.$ quartile +1.5 times the 843 interquartile range) and minimum ( $1^{\text {st }}$ quartile -1.5 times the interquartile range) values, 844 respectively. 
bioRxiv preprint doi: https://doi.org/10.1101/2021.07.26.453819; this version posted July 26, 2021. The copyright holder for this preprint (which

was not certified by peer review) is the author/funder, who has granted bioRxiv a license to display the preprint in perpetuity. It is made available under aCC-BY-NC-ND 4.0 International license.

845 Table 3 Total diversity statistics per species for captured loci.

\begin{tabular}{llllll}
\hline Species name & NKR & SNPs & SNPs/kbp & $\pi$ & $\boldsymbol{N}_{+}$ \\
\hline Alopecurus pratensis & 1.18 & 3,819 & 19.66 & $9.50 \times 10^{-3}$ & 234 \\
Arrbenatherum elatius & 0.78 & 287 & 5.29 & $2.66 \times 10^{-3}$ & 72 \\
Cynosurus cristatus & 1.03 & 1,040 & 7.3 & $3.35 \times 10^{-3}$ & 173 \\
Dactylis glomerata & 1.36 & 3,022 & 15.91 & $7.84 \times 10^{-3}$ & 229 \\
Festuca pratensis & 1.11 & 3,525 & 26.81 & $1.73 \times 10^{-2}$ & 188 \\
Festuca rubra & 0.76 & 3,208 & 14.77 & $7.15 \times 10^{-3}$ & 281 \\
Lolium multiflorum & 0.85 & 3,009 & 17.08 & $8.38 \times 10^{-3}$ & 218 \\
Lolium perenne & 0.65 & 1,479 & 8.55 & $5.05 \times 10^{-3}$ & 211 \\
Lotus corniculatus & 1.29 & 4,339 & 9.68 & $4.25 \times 10^{-3}$ & 477 \\
Medicago sativa & 1.20 & 5,615 & 12.47 & $5.38 \times 10^{-3}$ & 507 \\
Onobrychis viciifolia & 1.17 & 3,259 & 8.84 & $4.03 \times 10^{-3}$ & 416 \\
Pbleum pratense & 1.13 & 6,094 & 20.12 & $8.67 \times 10^{-3}$ & 354 \\
Poa pratensis & 1.24 & 5,723 & 17.4 & $9.28 \times 10^{-3}$ & 415 \\
Trifolium pratense & 1.31 & 2,766 & 6.36 & $2.76 \times 10^{-3}$ & 469 \\
Trifolium repens & 1.55 & 8,463 & 20.17 & $9.41 \times 10^{-3}$ & 536 \\
Trisetum flavescens & 0.53 & 696 & 4.88 & $4.92 \times 10^{-3}$ & 179 \\
\hline
\end{tabular}

$846+\boldsymbol{N}$ : Total quality-controlled loci captured per species. 
bioRxiv preprint doi: https://doi.org/10.1101/2021.07.26.453819; this version posted July 26, 2021. The copyright holder for this preprint (which

was not certified by peer review) is the author/funder, who has granted bioRxiv a license to display the preprint in perpetuity. It is made available under aCC-BY-NC-ND 4.0 International license.

847 Table 4 Median genetic diversity statistics of the selected loci.

\begin{tabular}{llllll}
\hline Locus & NKR & SNPs & SNPs/kbp & $\pi_{+}$ & $\boldsymbol{N}$ \\
\hline ortho-1390 & $1.00(0.84-1.36)$ & $9.50(5.50-18.00)$ & $16.00(6.66-27.32)$ & $8.09 \times 10^{-3}\left(2.45 \times 10^{-3}-1.21 \times 10^{-2}\right)$ & 15 \\
ortho-1347 & $1.20(0.79-1.35)$ & $22.00(6.00-31.00)$ & $22.00(6.83-34.66)$ & $8.65 \times 10^{-3}\left(4.15 \times 10^{-3}-1.09 \times 10^{-2}\right)$ & 15 \\
ortho-1318 & $1.40(1.06-1.60)$ & $16.50(8.50-35.00)$ & $26.46(9.80-39.14)$ & $9.68 \times 10^{-3}\left(4.29 \times 10^{-3}-1.95 \times 10^{-2}\right)$ & 14 \\
ortho-1262 & $1.42(1.13-1.66)$ & $16.50(13.62-22.25)$ & $28.13(19.12-31.58)$ & $9.94 \times 10^{-3}\left(7.27 \times 10^{-3}-1.50 \times 10^{-2}\right)$ & 16 \\
ortho-1211 & $1.43(0.93-2.13)$ & $14.00(9.50-21.00)$ & $19.71(10.70-29.00)$ & $6.36 \times 10^{-3}\left(5.59 \times 10^{-3}-8.63 \times 10^{-3}\right)$ & 16 \\
uce-11004576 & $1.49(1.12-1.82)$ & $9.00(2.50-14.50)$ & $9.55(3.38-26.15)$ & $3.42 \times 10^{-3}\left(1.33 \times 10^{-3}-8.68 \times 10^{-3}\right)$ & 14 \\
uce-11004158 & $1.68(1.20-2.10)$ & $24.00(11.50-31.00)$ & $27.06(18.69-38.19)$ & $1.03 \times 10^{-2}\left(8.50 \times 10^{-3}-1.75 \times 10^{-2}\right)$ & 15 \\
ortho-1288 & $1.74(1.28-1.97)$ & $36.00(14.25-62.75)$ & $39.82(15.76-68.32)$ & $2.10 \times 10^{-2}\left(9.09 \times 10^{-3}-3.49 \times 10^{-2}\right)$ & 12 \\
ortho-165 & $1.75(1.52-2.24)$ & $15.00(6.00-24.75)$ & $18.97(7.89-28.09)$ & $6.23 \times 10^{-3}\left(2.88 \times 10^{-3}-1.08 \times 10^{-2}\right)$ & 14 \\
ortho-1520 & $1.91(1.68-2.07)$ & $27.00(18.00-33.00)$ & $29.22(19.72-39.14)$ & $1.26 \times 10^{-2}\left(8.34 \times 10^{-3}-1.73 \times 10^{-2}\right)$ & 3 \\
uce-11001396 & $2.62(1.33-2.77)$ & $7.67(6.00-13.00)$ & $14.59(11.00-35.00)$ & $5.85 \times 10^{-3}\left(3.64 \times 10^{-3}-8.53 \times 10^{-3}\right)$ & 13 \\
\hline
\end{tabular}

$848 \uparrow \pi$ : nucleotide diversity per $1 \mathrm{kbp} ; \boldsymbol{N}$. number of species where a locus was found. 
bioRxiv preprint doi: https://doi.org/10.1101/2021.07.26.453819; this version posted July 26, 2021. The copyright holder for this preprint (which

was not certified by peer review) is the author/funder, who has granted bioRxiv a license to display the preprint in perpetuity. It is made available under aCC-BY-NC-ND 4.0 International license.

Table 5 Amplicon sequencing output summary.

\begin{tabular}{cccccccc}
\hline Family & Species name & Raw read pairs & $\begin{array}{c}\text { \% Merged } \\
\text { on-target }_{+}\end{array}$ & $\begin{array}{c}\text { \%Duplicates } \\
\text { in merged }\end{array}$ & Reads per amplicon & $\begin{array}{c}\text { Amplicons } \\
\text { per library }^{*}\end{array}$ & $\begin{array}{c}\text { Library } \\
\text { count }\end{array}$ \\
\hline Fabaceae & Trifolium pratense & $3,034,018$ & 49.67 & 96.32 & $6,684(4,119-15,068)$ & 8 & 19 \\
& Trifolium repens & $2,864,276$ & 37.42 & 95.99 & $5,947(2,935-11,129)$ & 7 & 19 \\
\hline Poaceae & Dactylis glomerata & $1,987,368$ & 28.79 & 91.34 & $2,652(1,254-5,476)$ & 7 & 19 \\
& Festuca pratensis & $2,250,146$ & 28.34 & 92.63 & $2,208(945-6,793)$ & 7 & 19 \\
& Lolium perenne & $2,306,113$ & 23.49 & 91.69 & $1,273(577-4,876)$ & $8(7,8)$ & 19 \\
\hline Fabaceae & All & $5,898,294$ & 43.72 & 96.18 & $6,446(3,336-13,948)$ & $7(7-8)$ & 38 \\
Poaceae & All & $\mathbf{6 , 5 4 3 , 6 2 7}$ & 26.77 & 91.92 & $1,901(868-5,870)$ & 7 & 57 \\
\hline & Total & $\mathbf{1 2 , 4 4 1 , 9 2 1}$ & 34.81 & 94.46 & $4,149(\mathbf{1 , 1 7 4 - 8 , 4 1 1 )}$ & $7(7-8)$ & 95 \\
\hline
\end{tabular}

$850 \uparrow$ Merged, on-target reads are reads that were quality-controlled and successfully merged and 851 mapped to a reference amplicon; percentage is in relation to raw read pairs. $¥$ Median and 852 interquartile range calculated considering only quality-controlled amplicons (e.g., amplicons with 853 five or more mapped reads). Interquartile range shown for groups that have variation. 
(a) 25
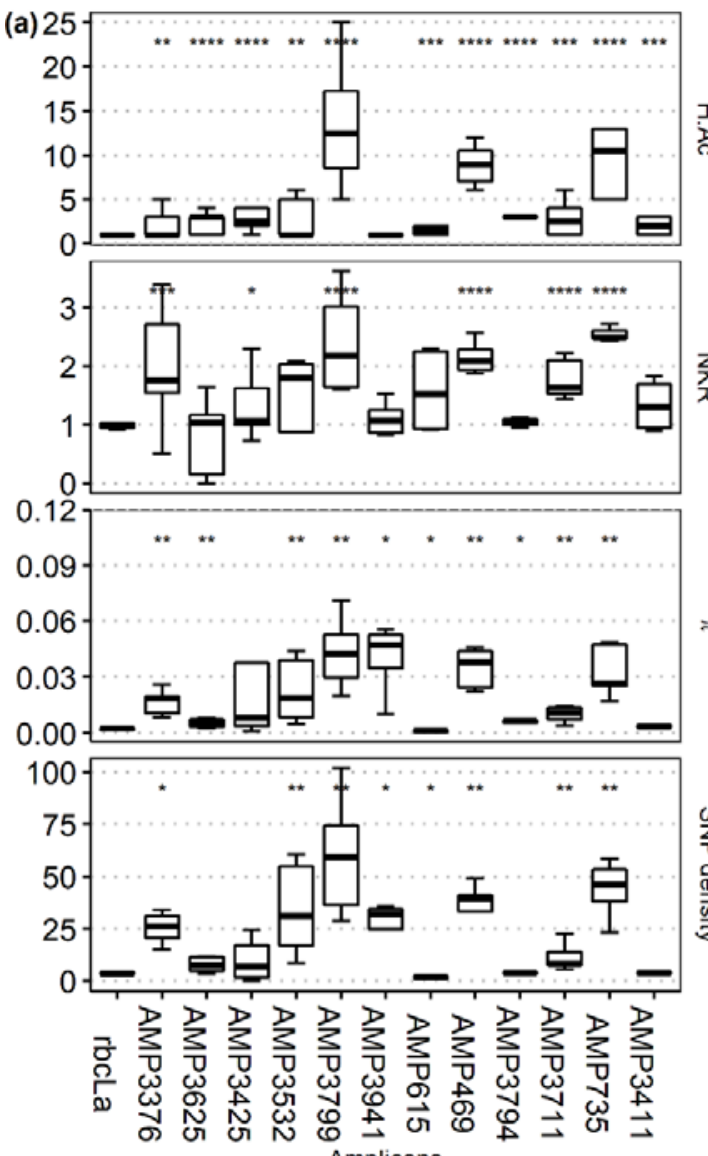

Amplicons (b)

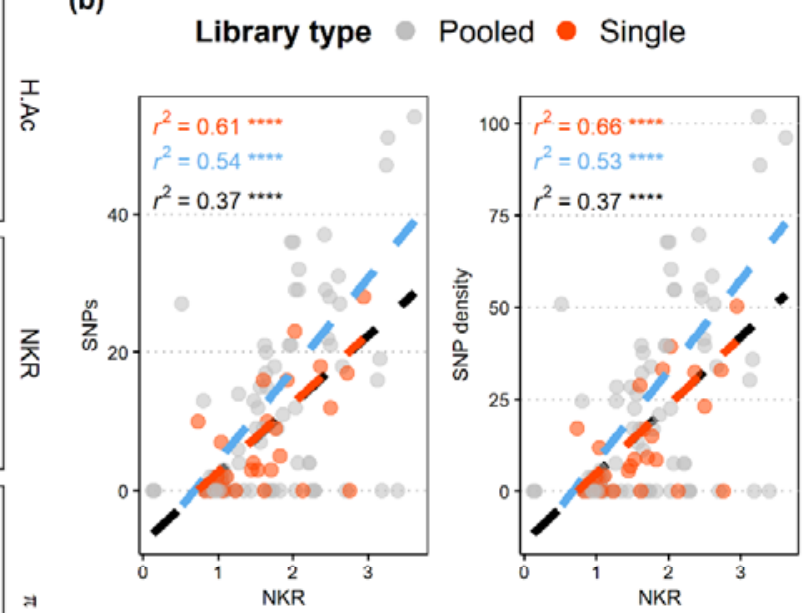

the
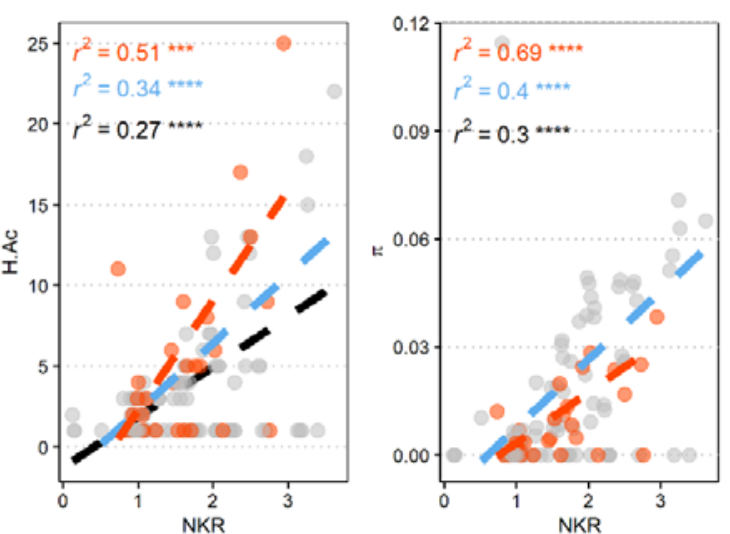

857

Figure 3 Sequence-based genetic diversity in groups of 16 plants per species. (a) SNPbased haplotype allele count (H.Ac), normalized $k$-mer richness (NKR), nucleotide diversity $(\pi)$ and SNP density (SNPs/kbp) at each locus in groups of sixteen plants per species. Amplicons without SNP calls were assumed to have only one haplotype allele in all sixteen plants. Wilcoxon test's significance levels are in relation to $r b c \mathrm{~L} a$. (b) Regression of diversity metrics per amplicon. Red dots show metrics from libraries generated from single-plant sequencing libraries; gray dots show metrics for libraries generated from pooled-plant sequencing libraries. Each dot represents one amplicon in a single- or pooled-plant libraries. The $\mathrm{r}^{2}$ value and $P$-value for all amplicons are shown in black; in blue, the same is shown only for amplicons with SNP calls; in red, only for amplicons from single-plant libraries and with SNP calls. Significance levels for $P$-values: 0.0001 $=* * * *, 0.001=* * *, 0.01=* *, 0.05=*$, ns $=$ non-significant. The upper and bottom ends of boxplots indicate the $3^{\text {rd }}$ and $1^{\text {st }}$ quartiles, respectively. The black line inside of boxplots indicates the median value. The upper and lower whiskers in boxplots indicate the maximum ( $3{ }^{\text {rd }}$ quartile +1.5 times the interquartile range) and minimum ( $1^{\text {st }}$ quartile -1.5 times the interquartile range) values, respectively. 
bioRxiv preprint doi: https://doi.org/10.1101/2021.07.26.453819; this version posted July 26, 2021. The copyright holder for this preprint (which

was not certified by peer review) is the author/funder, who has granted bioRxiv a license to display the preprint in perpetuity. It is made available under aCC-BY-NC-ND 4.0 International license.

872 Table 6 Genetic diversity per species based on the multispecies amplicons in single-plant

873 libraries

\begin{tabular}{llllllll}
\hline Species name & NKR & SNPs & SNPs/kbp & $\pi$ & H.Ac & Total length (bp) + & N \\
\hline Dactylis glomerata & 1.89 & 62 & 18.01 & $1.29 \times 10^{-2}$ & 40 & 3,442 & 6 \\
Festuca pratensis & 1.00 & 27 & 7.75 & $5.19 \times 10^{-3}$ & 19 & 3,483 & 6 \\
Lolium pereme & 1.41 & 44 & 12.61 & $8.49 \times 10^{-3}$ & 43 & 3,490 & 6 \\
Trifolium pratense & 1.42 & 36 & 10.28 & $7.63 \times 10^{-3}$ & 36 & 3,503 & 7 \\
Triffolium repens & 2.01 & 23 & $\mathbf{1 1 . 5 6}$ & $1.05 \times 10^{-2}$ & 13 & 1,989 & 5 \\
\hline Mean & 1.55 & 38.40 & $\mathbf{1 2 . 0 4}$ & $8.94 \times 10^{-3}$ & 30.20 & $3,181.40$ & 6.00 \\
\hline
\end{tabular}

874 Sum of the lengths of all amplicons in each species expressed in base pairs (bp). All metrics

875 exclude the DNA barcode $r b c \mathrm{a}$ and amplicon AMP469 for Trifolium repens. 
876 Table 7 Median genetic diversity statistics per amplicon including single- and pooled-

877 plant samples for the multispecies amplicons and DNA barcode $\boldsymbol{r} b \boldsymbol{c L}$.

\begin{tabular}{|c|c|c|c|c|c|c|c|}
\hline Amplicon & Locus & NKR & SNPs & SNPs/kbp & $\pi$ & H.Ac & $N_{+}$ \\
\hline rbcta & rbcla & $\begin{array}{l}0.98(0.95- \\
1.03)\end{array}$ & $2.00(2.00-2.00)$ & $3.71(3.58-3.77)$ & $\begin{array}{l}2.37 \times 10^{-3}\left(2.01 \times 10^{-3}-2.56 \times\right. \\
\left.10^{-3}\right)\end{array}$ & $\begin{array}{l}1.00(1.00- \\
1.00)\end{array}$ & 20 \\
\hline & & $1.04(0.15-$ & & $7.54(4.71-$ & $5.31 \times 10^{-3}\left(3.49 \times 10^{-3}-7.15 \times\right.$ & $3.00(1.00-$ & \\
\hline AMP3625 & $0-165$ & $\begin{array}{l}1.17) \\
1.05(1.00-\end{array}$ & $4.00(2.50-6.50)$ & 11.55) & $\begin{array}{l}\left.10^{-3}\right) \\
6.34 \times 10^{-3}\left(5.56 \times 10^{-3}-6.50 \times\right.\end{array}$ & $\begin{array}{l}3.00) \\
3.00(3.00-\end{array}$ & 12 \\
\hline AMP3794 & $\begin{array}{l}\text { U-1 } 1001396 \\
\text { U- }\end{array}$ & $1.10)$ & $2.00(2.00-2.00)$ & $3.77(3.77-3.86)$ & $\left.10^{-3}\right)$ & $3.00)$ & 4 \\
\hline AMP3425 & $\begin{array}{l}11004158 / \\
0-1519\end{array}$ & $\begin{array}{l}1.06(1.00- \\
1.63)\end{array}$ & $4.00(1.00-10.00)$ & $\begin{array}{l}6.91(1.71- \\
17.12)\end{array}$ & $\begin{array}{l}8.34 \times 10^{-3}\left(3.69 \times 10^{-3}-3.77 \times\right. \\
\left.10^{-2}\right)\end{array}$ & $\begin{array}{l}2.50(2.00- \\
4.00)\end{array}$ & 12 \\
\hline AMP3941 & O-1211 & $\begin{array}{l}1.07(0.86- \\
1.25)\end{array}$ & $\begin{array}{l}17.00(12.75- \\
18.25)\end{array}$ & $\begin{array}{l}32.05(24.79- \\
34.41)\end{array}$ & $\begin{array}{l}4.71 \times 10^{-2}\left(3.47 \times 10^{-2}-5.23 \times\right. \\
\left.10^{-2}\right)\end{array}$ & $1.00(1.00-$ & 20 \\
\hline & & $1.30(0.94-$ & & & $3.62 \times 10^{-3}\left(3.41 \times 10^{-3}-3.98 \times\right.$ & $2.00(1.00-$ & \\
\hline AMP3411 & $0-165$ & $1.70)$ & $2.00(2.00-2.00)$ & $3.77(3.77-3.79)$ & $\left.10^{-3}\right)$ & $3.00)$ & 8 \\
\hline AMP615 & $\mathrm{U}-11004576$ & $\begin{array}{l}1.53(0.93- \\
2.25)\end{array}$ & $1.00(1.00-1.00)$ & $1.89(1.83-1.89)$ & $\begin{array}{l}1.33 \times 10^{-3}\left(1.27 \times 10^{-3}-1.35 \times\right. \\
\left.10^{-3}\right)\end{array}$ & $\begin{array}{l}1.50(1.00- \\
2.00)\end{array}$ & 8 \\
\hline AMP3711 & $0-1390$ & $\begin{array}{l}1.63(1.52- \\
2.10)\end{array}$ & $4.00(3.75-7.50)$ & $\begin{array}{l}8.34(7.54- \\
14.14)\end{array}$ & $\begin{array}{l}1.10 \times 10^{-2}\left(7.20 \times 10^{-3}-1.36 \times\right. \\
\left.10^{-2}\right)\end{array}$ & $\begin{array}{l}2.50(1.00- \\
4.00)\end{array}$ & 8 \\
\hline AMP3376 & O-1347 & $\begin{array}{l}1.76(1.54- \\
2.71)\end{array}$ & $\begin{array}{l}14.00(11.50- \\
16.50)\end{array}$ & $\begin{array}{l}26.40(20.65- \\
31.11)\end{array}$ & $\begin{array}{l}1.87 \times 10^{-2}\left(1.06 \times 10^{-2}-1.96 \times\right. \\
\left.10^{-2}\right)\end{array}$ & $\begin{array}{l}1.00(1.00- \\
3.00)\end{array}$ & 12 \\
\hline AMP3532 & O-1288 & $\begin{array}{l}1.81(0.87- \\
2.03)\end{array}$ & $\begin{array}{l}17.50(9.00- \\
29.00)\end{array}$ & $\begin{array}{l}30.97(16.97- \\
54.68)\end{array}$ & $\begin{array}{l}1.88 \times 10^{-2}\left(8.23 \times 10^{-3}-3.88 \times\right. \\
\left.10^{-2}\right)\end{array}$ & $\begin{array}{l}1.00(1.00- \\
5.00)\end{array}$ & 12 \\
\hline AMP469 & $0-1262$ & $\begin{array}{l}2.09(1.94- \\
2.28)\end{array}$ & $\begin{array}{l}21.00(16.00- \\
21.75)\end{array}$ & $\begin{array}{l}39.60(33.20- \\
41.01)\end{array}$ & $\begin{array}{l}3.78 \times 10^{-2}\left(2.44 \times 10^{-2}-4.38 \times\right. \\
\left.10^{-2}\right)\end{array}$ & $\begin{array}{l}9.00(7.00- \\
10.50)\end{array}$ & 8 \\
\hline AMP3799 & O-1318 & $\begin{array}{l}2.18(1.64- \\
3.02)\end{array}$ & $\begin{array}{l}32.00(19.50- \\
39.50)\end{array}$ & $\begin{array}{l}59.12(36.38- \\
74.48)\end{array}$ & $\begin{array}{l}4.25 \times 10^{-2}\left(2.97 \times 10^{-2}-5.27 \times\right. \\
\left.10^{-2}\right)\end{array}$ & $\begin{array}{l}12.50(8.50- \\
17.25)\end{array}$ & 12 \\
\hline AMP735 & O-1520 & $\begin{array}{l}2.50(2.48- \\
2.61)\end{array}$ & $\begin{array}{l}24.50(20.00- \\
28.25)\end{array}$ & $\begin{array}{l}46.20(37.92- \\
53.27)\end{array}$ & $\begin{array}{l}2.68 \times 10^{-2}\left(2.55 \times 10^{-2}-4.73 \times\right. \\
\left.10^{-2}\right)\end{array}$ & $\begin{array}{l}10.50(5.00- \\
13.00)\end{array}$ & 8 \\
\hline
\end{tabular}

$878+$ The number of diversity estimates considered for the median and interquartile range 879 calculations. For each amplicon, there are maximum four estimates per species: one from single880 plant samples and three from pooled-plant samples.

881 

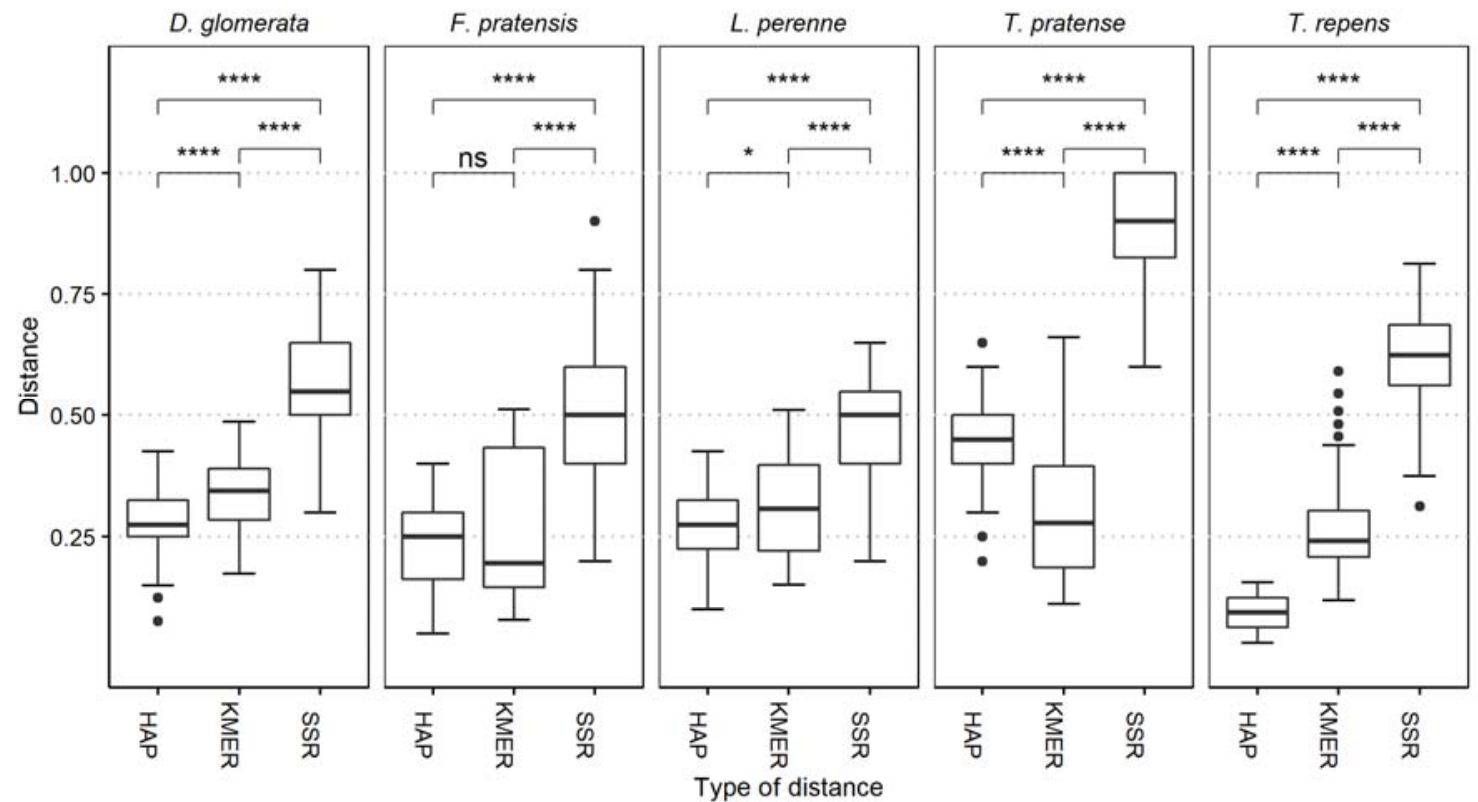

882

883

Figure 4 Comparison of pairwise distances derived from single-plant samples using

884 sequence- and SSR-based genetic diversity statistics. Prevosti's distance was used for amplicon haplotype-based multi-locus genotypes (HAP-MLGs) and SSR-based MLGs (SSRMLGs). Sourmash-derived distance was used for $k$-mers. Five amplicons per species (four, in case of T. repens) were used to calculate distance matrices for HAP-MLGs and k-mers. The five most polymorphic SSRs per species were used to calculate the SSR-MLGs distance matrix. Samples with $>5 \%$ missing loci were removed. Wilcoxon test's significance levels are shown for all possible comparisons. Significance levels for $P$-values: $0.0001=* * * *, 0.001=* * *, 0.01=* *$, $0.05=*$, ns $=$ non-significant. The upper and bottom ends of boxplots indicate the $3^{\text {rd }}$ and $1^{\text {st }}$ quartiles, respectively. The black line inside of boxplots indicates the median value. The upper and lower whiskers in boxplots indicate the maximum $\left(3^{\text {rd }}\right.$ quartile +1.5 times the interquartile range) and minimum ( $1^{\text {st }}$ quartile -1.5 times the interquartile range) values, respectively. Dots on top and at the bottom of boxplots indicate outliers. 
bioRxiv preprint doi: https://doi.org/10.1101/2021.07.26.453819; this version posted July 26, 2021. The copyright holder for this preprint (which

was not certified by peer review) is the author/funder, who has granted bioRxiv a license to display the preprint in perpetuity. It is made available under aCC-BY-NC-ND 4.0 International license.

897 Table 7. Median ratios between SSR- and amplicon sequencing-based multi-locus 898 pairwise distances.

\begin{tabular}{|c|c|c|c|c|c|c|c|}
\hline Family & Species name & Amplicons & SSRs & SSR:k-mer & SSR:HAP & k-mer:HAP & $N$ \\
\hline \multirow{15}{*}{ Poaceae } & & AMP3376 & DG004 & & & & \\
\hline & & AMP3425 & DG006 & & & & \\
\hline & & AMP3532 & DG010 & & & & \\
\hline & & AMP3625 & DG011 & & & & \\
\hline & Dactylis glomerata & AMP3799 & DG012 & $1.68(1.33-2.10)$ & $2.16(1.67-2.62)$ & $1.23(1.04-1.51)$ & 120 \\
\hline & & AMP3376 & FA49 & & & & \\
\hline & & AMP3425 & LM26 & & & & \\
\hline & & AMP3532 & LM29 & & & & \\
\hline & & AMP3625 & LP27 & & & & \\
\hline & Festuca pratensis & AMP3799 & LP47 & $2.62(1.17-3.44)$ & $2.00(1.62-2.67)$ & $0.93(0.63-1.49)$ & 66 \\
\hline & & AMP3376 & LP07 & & & & \\
\hline & & AMP3425 & LP20 & & & & \\
\hline & & AMP3532 & LP23 & & & & \\
\hline & & AMP3625 & LP13 & & & & \\
\hline & Lolitum perenne & AMP3799 & LP16 & $1.68(1.22-2.31)$ & $1.85(1.48-2.20)$ & $1.21(0.80-1.52)$ & 66 \\
\hline \multirow{10}{*}{ Fabaceae } & & AMP3411 & TP45 & & & & \\
\hline & & AMP3711 & ТP46 & & & & \\
\hline & & AMP615 & TP50 & & & & \\
\hline & & AMP735 & TP34 & & & & \\
\hline & Trifolium pratense & AMP469 & TP10 & $3.32(2.12-4.98)$ & $2.00(1.78-2.25)$ & $0.68(0.39-0.93)$ & 66 \\
\hline & & AMP3411 & TR04 & & & & \\
\hline & & AMP3711 & TR09 & & & & \\
\hline & & AMP615 & TR10 & & & & \\
\hline & Trifolitum repens & AMP735 & TR13 & $2.42(1.88-3.12)$ & $7.33(5.50-10.00)$ & $3.31(2.16-4.62)$ & 115 \\
\hline & Total & & & $2.07(1.45-2.93)$ & $2.36(1.80-4.50)$ & $1.29(0.88-2.20)$ & 433 \\
\hline
\end{tabular}

899 